\title{
Reconciling Elastic and Equilibrium Methods for Static Analysis
}

\author{
HIJUNG V. SHIN and CHRISTOPHER F. PORST \\ Massachusetts Institute of Technology \\ ETIENNE VOUGA \\ The University of Texas at Austin \\ and \\ JOHN OCHSENDORF and FRÉDO DURAND \\ Massachusetts Institute of Technology
}

\begin{abstract}
We examine two widely used classes of methods for static analysis of masonry buildings: linear elasticity analysis using finite elements and equilibrium methods. It is often claimed in the literature that finite element analysis is less accurate than equilibrium analysis when it comes to masonry analysis; we examine and qualify this claimed inaccuracy, provide a systematic explanation for the discrepancy observed between their results, and present a unified formulation of the two approaches to stability analysis. We prove that both approaches can be viewed as equivalent, dual methods for getting the same answer to the same problem. We validate our observations with simulations and physical tilt experiments of structures.
\end{abstract}

Categories and Subject Descriptors: I.3.5 [Computer Graphics]: Computational Geometry and Object Modeling_Physically based modeling

General Terms: Algorithms, Experimentation, Theory

Additional Key Words and Phrases: Static equilibrium analysis, finite element method, limit-state analysis, masonry analysis, self-supporting masonry, contact constraints

ACM Reference Format:

Hijung V. Shin, Christopher F. Porst, Etienne Vouga, John Ochsendorf, and Frédo Durand. 2016. Reconciling elastic and equilibrium methods for static analysis. ACM Trans. Graph. 35, 2, Article 13 (February 2016), 16 pages. DOI: http://dx.doi.org/10.1145/2835173

\section{INTRODUCTION}

The design of self-supporting masonry is an ancient and elegant technique that combines the form and function of geometry. The

This project is partially funded by NSF DMS-1304211 and Royal Dutch Shell. Hijung V. Shin acknowledges Samsung Scholarship for their support. Authors' addresses: H. V. Shin; email: hishin@mit.edu; C. F. Porst; email: cporst14@gmail.com; E. Vouga; email: evouga@cs.utexas.edu; J. Ochsendorf; email: jao@mit.edu; F. Durand; email: fredo@mit.edu.

Permission to make digital or hard copies of all or part of this work for personal or classroom use is granted without fee provided that copies are not made or distributed for profit or commercial advantage and that copies bear this notice and the full citation on the first page. Copyrights for components of this work owned by others than the author(s) must be honored. Abstracting with credit is permitted. To copy otherwise, or republish, to post on servers or to redistribute to lists, requires prior specific permission and/or a fee. Request permissions from permissions@acm.org.

2016 Copyright is held by the owner/author(s). Publication rights licensed to ACM.

0730-0301/2016/02-ART13 $\$ 15.00$

DOI: http://dx.doi.org/10.1145/2835173 stability of a structure depends directly on its geometry rather than relying on the strength of reinforcement materials such as steel. Architects and engineers have explored the link between geometry and mechanics since antiquity, and recent computer graphics approaches have expanded this endeavor and created design tools that are informed by or optimized for structural goals (e.g. Deuss et al. [2014], Whiting et al. [2012], Panozzo et al. [2013], Liu et al. [2013], Vouga et al. [2012], and Block [2009]). This article considers masonry structures - those made of unreinforced stone or brick, like the Aqueduct of Segovia or Gothic cathedrals - that are stable under their own weight. Masonry has several well-accepted characteristics: it is extremely strong in compression, and for practical purposes its compressive strength can be assumed to be infinite [Heyman 1966]. When a masonry structure fails, usually it is not due to material failure, but to geometric failure: blocks hinge apart from each other and topple. Sometimes, mortar is placed between block interfaces, but mortar is structurally weak and susceptible to cracking and weathering, so we assume that mortar does not contribute to the stability of the structure-that there is no tensile capacity in between blocks.

The best approach for assessing the structural stability of masonry design remains a subject of debate. Given the success of the Finite Element Method (FEM) for strength analysis in reinforced structures and the wide availability of software tools [Autodesk 2014; Computers and Structures, Inc. 2014; Dassault Système 2014], practitioners have attempted to apply it to traditional masonry designs. They use linear elastic analysis to predict whether a structure will stand under certain loads, either by directly measuring displacements of blocks or by measuring indicators of instability such as tension at block interfaces, with little success. Consider the Aqueduct of Segovia, the most prominent Roman monument left on the Iberian peninsula, which has stood for nearly two millennia (see Figure 1, top). Bravo et al. [1994] modeled the aqueduct assuming a homogeneous and isotropic material and neglecting the effect of masonry joints between blocks. When they analyzed the stress state of the pillars using FEM, they could not conclusively demonstrate that the aqueduct is stable under gravity without tension forces. Similarly, Block [2009] and Whiting [2012] remarked that using conventional FEM on masonry arch modeled as a single continuous structure, they could not distinguish between stable and unstable circular arches (see Figure 1, bottom). Instead, they advocated a different type of approach based on direct analysis of force equilibrium (discussed later), which can reliably predict whether a structure is stable or not without computing the elastic deformation of materials.

Although it is expected that naïvely modeling a structure as a single continuum and applying linearized FEM is inadequate to analyze masonry, this is the method employed by many practitioners, 

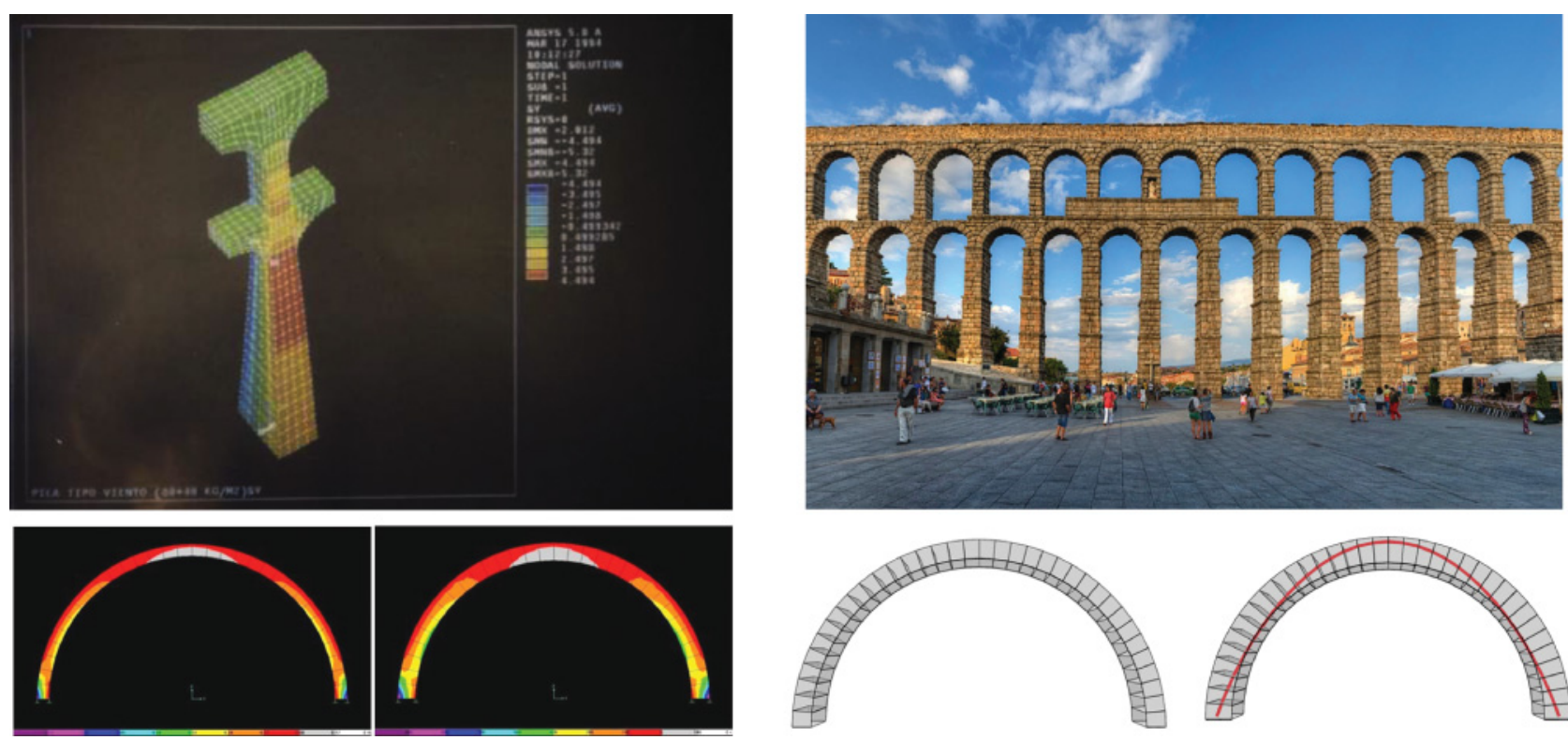

Fig. 1. The Aqueduct of Segovia, a masonry structure that has been standing since antiquity (top, right). Finite element analysis of a section of the aqueduct does not conclusively demonstrate its stability (top, left, image from Bravo et al. [1994]). Similarly, linear elastic FEM fails to differentiate between the stability of an infeasible arch that is too thin to stand on its own (bottom, leftmost) and a thicker, feasible arch (bottom, left) (image from Block et al. [2006]). In contrast, equilibrium methods correctly predict that the thin arch is infeasible (bottom, right) and that the thick arch is feasible (bottom, rightmost).

partly due to the lack of alternatives in commercial software. On the other hand, in the broader context, FEM has been tremendously successful at numerically simulating a variety of physical systems in engineering, biology, mechanics, and medicine. So, it seems plausible that with proper assumptions and material models, FEM should be able to correctly analyze masonry structures. Is there a better way to apply FEM to masonry structures? Certainly we would expect a full dynamics simulation of the masonry, using complex nonlinear material and contact models and very fine spatial and temporal discretization, to correctly predict the behavior of masonry, albeit at a tremendous computational cost. What are the necessary modifications to continuum, linearized FEM to obtain accurate results for masonry? In other words, what is its primary source of inaccuracy? Several possible explanations are cited, but to our knowledge no systematic examination exists for which of these are important sources of error and which are not:

-It is possible for masonry structures to hinge while still remaining stable. Modeling the entire masonry as a single continuous structure does not reflect the contact conditions at the block interfaces.

- Simulating accurate stresses in the structure requires choosing a correct constitutive model for the stone blocks. The simplest choice of constitutive model - a linear stress-strain relationship and a linearized strain measure-is not correct for stone.

- Similarly, stone is extremely stiff, and its high Young's modulus potentially introduces numerical stability issues during simulation.

-Most analysis assumes that the shape and positions of the blocks, specified as input by the user, are initial conditions, and then solve for the final deformation of the structure. In other words, stability is analyzed using a forward simulation. The final, deformed geometry is not the same as the input geometry. This can be a problem when we are interested in the stability of the input structure as is, without further deformation.
Table I. Comparison of the FEM Versus Equilibrium Methods

\begin{tabular}{c|cc}
\hline Method & FEM & Equilibrium \\
\hline Solution variable & Deformation & Force \\
Material & Elastic & Rigid \\
Geometry & Deforms & Fixed \\
\hline
\end{tabular}

- The expected stresses in a building are impossible to determine from the geometry alone, due to static indeterminacy. Many possible sets of internal stresses are consistent with the observed state of the building, and slight changes in the environment can cause large changes in measured stresses. An everyday example of this indeterminacy is a four-legged stool resting on a rigid, level floor: the stool is stable with only three of the four legs carrying the load, and which legs carry how much load can change drastically for even infinitesimal changes in the lengths of the legs. Finite element analysis necessarily solves for only one possible set of equilibrium stresses, and this solution is highly sensitive to initial conditions.

Equilibrium methods have been proposed as alternatives to finite element analysis to sidestep the aforementioned challenges. This line of attack can be traced back to the seminal work of Heyman [1966]. Equilibrium methods, also called limit analysis, recently became popular in the graphics community as an efficient way to guarantee stability of procedurally generated or interactively designed architecture [Whiting et al. 2009; Vouga et al. 2012; Panozzo et al. 2013]. The key idea in these methods is to ignore the elastic deformation of the stone entirely, and instead to treat the structure as piecewise rigid. The unknowns in these methods are the forces acting on these rigid pieces, rather than their displacements. Table I summarizes the key differences between finite-element- and equilibrium-based methods. (Equilibrium methods and FEM are surveyed in more detail in Sections 3 and 4, respectively.) Generally 
speaking, equilibrium methods are known to predict the stability of structures more reliably, but they are restricted to certain simple classes of masonry structure. So, how is it that equilibrium analysis obtains reliable results even without sophisticated modeling parameters? What is the source of discrepancy between linear analysis using FEM and equilibrium analysis?

In this article, we examine both classes of methods and seek an explanation for the discrepancy between their results. In particular, we rigorously evaluate the claim that equilibrium methods more reliably predict stability than finite elements. We expose the primary source of difficulty in using linear elastic FEM to analyze masonry, namely, that it does not correctly account for the geometric and topological nonlinearity of hinging and breaking contact at the block interfaces, and that, moreover these problems can persist even when the analysis is augmented with proper one-sided contact constraints. Finally, we propose a small modification to the FEM that avoids these problems and demonstrate that with this adjustment and proper interpretation of the FEM, both approaches to stability analysis can be viewed as equivalent, dual methods for getting the same answer to the same problem. Consequently, both equilibrium and finite-element methods can be used to reliably predict the stability of masonry, even with very simple and efficient models of the structure's material and geometry. We validate this observation by comparing the simulation results of both approaches to known analytic solutions, the commercial finite-element Abaqus package, and data from physical tilt tests. Both equilibrium and modified finite-element methods correctly predict the maximum tilt angle of structures, agreeing with analytical solutions and physical tests.

\section{RELATED WORK}

\subsection{Equilibrium Versus Elastic Approach}

The analysis of masonry structures has been a topic of study from as early as the 17th century [Huerta 2008] and can be divided into two schools of thought: the equilibrium approach and the elastic approach. We refer the reader to Heyman [1995], Huerta [2001], and Roca [2010] for an extensive historical overview.

The purpose of the equilibrium approach is to demonstrate whether a static state of equilibrium is possible. That is, can the forces and torques acting on each rigid block be balanced using compressive forces alone? In $2 \mathrm{D}$, the interaction between gravity and the contact forces acting on each block can be elegantly summarized using graphical methods. Force polygons translate equilibrium (force and torque balance) into the requirement that forces acting on a block link together to form a closed polygon. Thrust lines connect the resultant force at the interface between pairs of blocks, and the compression-only constraint requires that the thrust line go through the interior of the interface (Figure 2).

In 1748, Poleni used thrust line analysis to demonstrate the equilibrium of the cracked masonry dome of St. Peter's in Rome [Heyman 1995]. The foundation for the modern equilibrium approach was formally laid by Heyman [1966]. Under certain physical assumptions about masonry, Heyman's Safe Theorem of limit analysis posits: if it is possible to find an internal system of forces in equilibrium with the loads that does not violate the yield condition of the material, the structure will not collapse and it is "safe." This Safe Theorem is the basis of several recent computational algorithms, discussed later, for determining or optimizing the stability of masonry structures. The failure modes unveiled using equilibrium methods tend to be hinging and correspond to failures of the geometry of the structure rather than material failures (Figure 3).
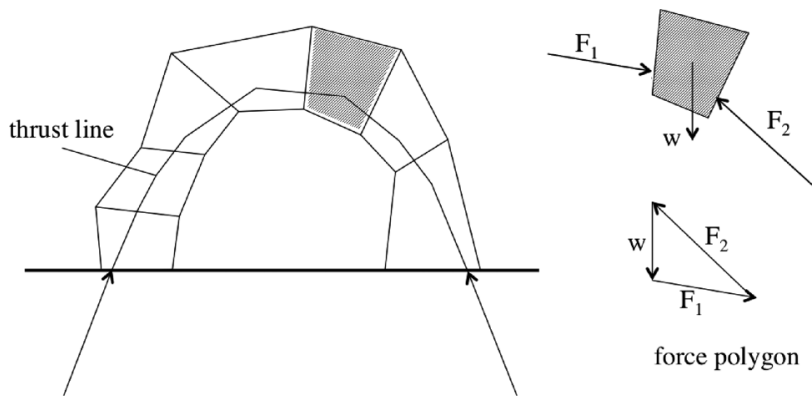

Fig. 2. The static equilibrium of an arch and a voussoir is demonstrated using a thrust line and a force polygon. The thrust line represents the path connecting the resultant compressive forces acting at the block interfaces. For the structure to be in equilibrium, there must be a line of thrust contained entirely within the section. The force polygon is constructed by linking the forces acting on a single block. A closed force polygon indicates that the block is in equilibrium. (Figure modified from Block and Ochsendorf [2007].)
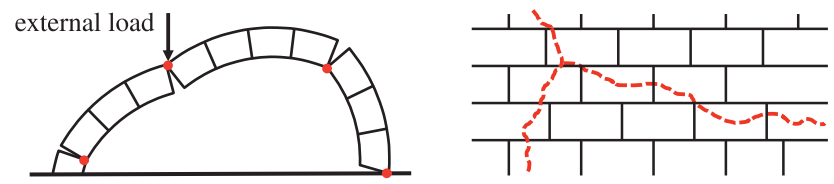

Fig. 3. A four-hinge collapse mechanism of an arch subject to a point load. The voussoirs rotate about their edge forming hinges, and eventually causing geometric failure (left). Cracks developing on a wall causing material failure $(r i g h t)$. Equilibrium methods focus on geometric failures, whereas elastic methods concern material failures.

In particular, equilibrium analysis is independent of the density or elastic modulus of the material (forces simply scale linearly).

Whereas equilibrium methods assume rigid geometry and focus on the balance of the external forces acting on each block, elastic analysis considers the internal stress and strain, and relates forces (and stress) to the displacement or deformation of the structure. Early, noncomputational work pioneering this idea in architecture includes the photo-elastic method of Mark [1982], which estimates the stress distributions on plane sections of cathedrals.

In elastic analysis, the behavior of the material is modeled mathematically, often using a linearized elastic approximation, and the elastic energy of the structure is expressed as a function of the displacement of each point of the structure. The configurations in static equilibrium are those that minimize the energy (with external forces taken into account via the principle of virtual work), or equivalently, those for which all forces balance at every point. Elastic analysis is often used to identify potential material failures when stress is too high, as opposed to the geometric failures found by equilibrium methods. Because of the difficulty in analytically solving the equilibrium equations, elastic analysis was initially limited to simple geometry [Huerta 2001]. In the 1970s, with the development of the FEM and increase in computational capabilities, elastic analysis via FEM became one of the most powerful and ubiquitous techniques in engineering. It is widely used for analyzing structures with both tensile and compressive forces, such as steel or reinforced concrete buildings. While FEM refers to a wide family of methods for solving PDEs over discretized function spaces and need not involve elastic analysis specifically, in this article, we use the term "FEM," in contrast to "equilibrium" methods, as a shorthand for the class of primal methods that analyze masonry stability by solving for 
displacements of elements in the structure using a linear stressstrain relationship.

\subsection{Physics-Based Simulation}

Many techniques [Martin et al. 2010; Xu et al. 2009; Terzopoulos et al. 1987; Terzopoulos and Fleischer 1988] use simulation to model geometry with physical constraints, but these approaches seek to compute realistic deformations rather than answer questions about static stability.

Modeling contact and friction accurately is especially important to simulating objects at rest. Contact and friction resolution has been recognized as a difficult problem. For instance, it is well known that rigid body dynamics with both contact and Coulomb friction is inconsistent [Painlevé 1895]. Recent strides in graphics toward incorporating realistic frictional contact into rigid body dynamics include a staggered algorithm for solving contact and friction together [Kaufman et al. 2008] with the goal of robustly simulating complex stacks of rigid and deformable bodies, adding contact constraints and friction to continuum-based models [Narain et al. 2010] to convincingly capture the complex behaviors of granular flow, or using shock propagation with an impulsebased [Guendelman et al. 2003] or velocity-based [Erleben 2007] contact solver to simulate stable stacking. However, these works focus primarily on dynamic simulations, whereas we are interested in static analysis. Hsu et al. [2012] use static equilibrium analysis to simulate large-scale stacking behavior, but they are interested in creating plausible animation rather than studying physical stability.

FEM and its variations are widely used in computer modeling and animation. Most relevant to our work are recent techniques by Chen et al. [2014] and Coros et al. [2012] on computing the elastic inverse problem: finding the rest shape of an elastic object so that the object deforms into a desired input shape by the given external forces. However, the former is targeted at 3D printing with elastic materials, and the latter deals with dynamic simulations rather than statics.

\subsection{Structural Optimization Using Static Analysis}

Static analysis has been applied to a wide range of areas with the goal of designing physically realistic objects. For example, static equilibrium has been used to determine plausible character poses [Shi et al. 2007] and realistic tree structures [Hart et al. 2003].

Several applications have proposed structural optimization targeted at fabrication. Umetani et al. [2012] developed a guided modeling interface for interactive design of wood furniture. Prevost et al. [2013] implemented stability objectives in order to print $3 \mathrm{D}$ models that stand upright without requiring an oversized base or additional stilts. FEM has been applied to strengthen 3D input objects [Stava et al. 2012] and to find worst-case loading scenarios [Zhou et al. 2013]. Umetani et al. [2010] presented real-time FEM analysis for interactive geometric modeling of objects such as metallophones.

In the context of building structures, Smith et al. [2002] applied statics to automatic truss design that optimizes for minimal material consumption. Recently, a number of works appeared in the graphics community focusing specifically on the design of structurally sound masonry, for instance, integrating static analysis with procedural modeling [Whiting et al. 2009], a method that was extended by Whiting et al. [2012] to generic quad meshes. Several methods [Vouga et al. 2012; Panozzo et al. 2013; de Goes et al. 2013; Liu et al. 2013] optimize structures based on the Thrust Network Analysis technique introduced by Block et al. [2007]. All of these works are in the tradition of equilibrium analysis.
Table II. Notation

\begin{tabular}{ccc}
\hline Symbol & Means & Size $^{*}$ \\
\hline$\tilde{\mathbf{x}}$ & Known ("input") quantities & \\
$\mathbf{x}_{\mathrm{aft}}$ & Geometry after deformation & $n \times 1$ \\
$\mathbf{x}_{\mathrm{bfr}}$ & Geometry before deformation & $n \times 1$ \\
$\mathbf{K}$ & Stiffness matrix & $n \times n$ \\
$\mathbf{f}_{\mathrm{in}}$ & Internal forces from elastic deformation & $\dagger$ \\
$\mathbf{f}_{\mathrm{W}}$ & Self-weight \& external applied forces & $\dagger$ \\
$\mathbf{f}_{\mathrm{ct}}$ & Contact forces between adjacent blocks & $\dagger$ \\
$\mathbf{n}^{i}$ & Normal direction of interface & $3 \times 1$ \\
$\mathbf{r}$ & Torque arm & $3 \times 1$ \\
$*_{n}=$ number ofelement nodes.
\end{tabular}

${ }^{*} n=$ number of element nodes.

${ }^{\dagger}$ Forces are discretized at different points of application, indicated by superscripts: $n$ (element nodes), $i$ (block interfaces), $b$ (block). Vector size depends likewise on the superscript.

${ }_{\ddagger}^{\ddagger}$ Finite element method only.

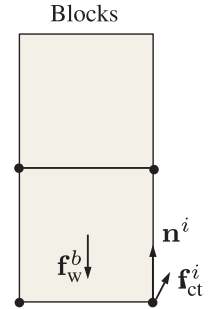

(a) Equilibrium method

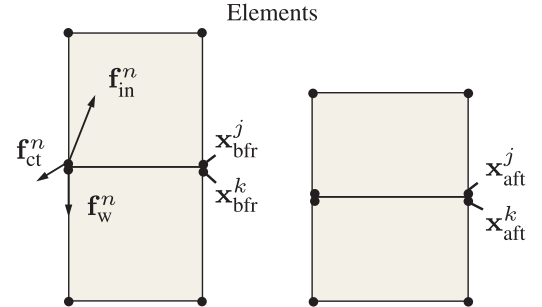

(b) Finite element method before / after deformation
Fig. 4. Variables used in the block equilibrium (Section 3) and finite element methods (Section 4). In the former, force and torque equilibrium is computed per block $(b)$, and contact forces $\mathbf{f}_{\mathrm{ct}}^{i}$ act at discrete contact nodes on the interfaces $(i)$ between blocks. In the latter, deformation occurs at element nodes $(n)$, where each element can be a subpart of a block. The deformation in turn induces elastic forces $\mathbf{f}_{\mathrm{in}}^{n}$ at the nodes. Contact constraints are formulated as functions of nodal positions (x), and consequently contact forces $\left(\mathbf{f}_{c t}^{n}\right)$ act also at the nodes.

Somewhat related to our goal of reconciling the equilibrium and FEM traditions, Fraternali [2010] and de Goes et al. [2013] derived correspondences between Thrust Networks and finite element discretization of stress functions, in the case of masonry with shell topology. We discuss in detail the relationship between Thrust Network Analysis, equilibrium of blocks, and FEM in the appendix.

\section{EQUILIBRIUM METHODS}

In what follows, we describe the equilibrium method and the FEM formulation and discuss their similarities and differences. We begin with the equilibrium method, which is known to predict the stability of masonry structures with high reliability, and is favored by researchers as a powerful yet simple alternative to the FEM for masonry analysis.

Please refer to Table II and Figure 4 for our notation and variables.

Assumptions. In contrast to elastic approaches, equilibrium methods assume that stones are infinitely stiff and strong. In addition, the method makes Heyman's three assumptions about the physics of masonry [1995]:

(1) Masonry has no tensile strength.

(2) It can resist infinite compression.

(3) Sliding failure does not occur within the masonry. 
Equilibrium methods rely on Heyman's Safe Theorem: if there exists an internal system of forces that are in equilibrium with the external loads (including self-weight) and that satisfy the previous assumptions, the structure will stand. Notice that the Safe Theorem guarantees stability if we can find any equilibrium state-we don't have to find or prove anything about the "actual" state of the stresses within the structure (which in any case can change discontinuously for small perturbations of the structure's geometry or loads, due to static indeterminacy). Equilibrium methods therefore check computationally for the existence of any possible equilibrium state.

In what follows, we describe one simple example of an equilibrium method, introduced by Whiting et al. [2012], for analyzing the stability of masonry structures built from polyhedral blocks (which we will call the "block equilibrium method"). Other equilibrium approaches, such as Block et al.'s Thrust Network Analysis [2009] and the improvements described in recent graphics papers (see Section 2.3) also rely on the Safe Theorem but are tailored to analyzing structures with the geometry of a thin surface, rather than a block network. Here, we will focus on the simple and general block equilibrium method rather than these surface-based methods, as the connection between it and finite elements will turn out to be particularly simple.

Static Equilibrium Condition. In order for a structure to stand in static equilibrium, it is required that the net force and net torque acting on the center of mass of each block $b$ equal zero, including the contact forces $\left(\mathbf{f}_{\mathrm{ct}}^{b}\right)$ and any forces arising from self-weight and externally applied loads $\left(\mathbf{f}_{\mathrm{w}}^{b}\right)$ :

$$
\begin{array}{r}
\sum_{i \in b} \mathbf{f}_{\mathrm{ct}}^{i}+\tilde{\mathbf{f}}_{\mathrm{w}}^{b}=\mathbf{f}_{\mathrm{ct}}^{b}+\tilde{\mathbf{f}}_{\mathrm{w}}^{b}=0 \quad \forall b \\
\sum_{i \in b}\left(\mathbf{f}_{\mathrm{ct}}^{i} \times \tilde{\mathbf{r}}_{\mathrm{ct}}^{i}\right)+\left(\tilde{\mathbf{f}}_{\mathrm{w}}^{b} \times \tilde{\mathbf{r}}_{\mathrm{w}}^{b}\right)=0 \quad \forall b,
\end{array}
$$

where $\mathbf{r}_{\mathrm{w}}^{b}$ is the torque arm with respect to the block's center of mass. A three-dimensional force $\mathbf{f}_{\mathrm{ct}}^{i}$ is positioned at a finite subset of vertices on the interface. Assuming a linear force distribution across the surface, three contact points per interface are sufficient to represent the force distribution. In our implementation, we follow Whiting et al. [2009] and place the forces on each vertex of the polygon that defines the interface between two blocks (overlapping region between the adjacent block faces). The assumption that masonry can resist infinite compression but no tension means that the contact forces at the interfaces between blocks must be compressive. This is expressed as an inequality constraint for each interface contact force:

$$
\mathbf{f}_{\mathrm{ct}}^{i} \cdot \mathbf{n}^{i} \geq 0
$$

where $\mathbf{n}^{i}$ is the interface normal pointing into the block (i.e., the direction of compression).

In addition to the equilibrium constraints, friction constraints can be approximated by constraining the tangential component of the interface forces $\left(\mathbf{I}-\mathbf{n}^{i} \mathbf{n}^{i} \boldsymbol{T}\right) \cdot \mathbf{f}_{\mathrm{ct}}^{i}$ to be within a conservative friction pyramid of their normal component, $\left(\mathbf{n}^{i} \mathbf{n}^{i \top}\right) \cdot \mathbf{f}_{\mathrm{ct}}^{i}$ :

$$
\left|\left(\mathbf{I}-\mathbf{n}^{i} \mathbf{n}^{i \top}\right) \cdot \mathbf{f}_{\mathrm{ct}}^{i}\right| \leq \alpha\left|\mathbf{f}_{\mathrm{ct}}^{i} \cdot \mathbf{n}^{i}\right|,
$$

where $\alpha$ is the coefficient of static friction. In our implementation, we assume infinite friction $(\alpha=\infty)$, so that all failure is caused by hinging and not sliding.

Force Solution. According to the Safe Theorem, if a feasible solution $\mathbf{f}_{\mathrm{ct}}$ exists that satisfies the aforementioned constraints, the structure will stand. Due to static indeterminacy, if a solution exists, that solution is typically far from unique. (Consider the case of the four-legged stool mentioned earlier.) To facilitate numerically solving for a solution, or to choose a particularly intuitive set of forces for visualization, often a linear or quadratic objective function (e.g., Whiting et al. [2009] uses weighted sum-of squares) is included and the problem is solved as a linear or quadratic program with linear constraints. Note that the solution $\mathbf{f}_{\mathrm{ct}}$ is not intended to represent the actual state of the forces in the structure. The key to stability is the existence of a feasible solution, and not the solution itself.

To summarize, in the equilibrium method, we take an input geometry of a structure and formulate a linear program (LP) or a quadratic program (QP) that enforces equilibrium constraints [Livesley 1978]. The requirement that forces must be compressive is a first-class citizen, included in the constraints. If a solution exists, the structure is stable. One beauty of the method is that it handles contact constraints and hyperstatic equilibrium naturally: by treating the blocks as rigid, contact constraints - that blocks can hinge but cannot interpenetrate - are implicitly satisfied, and by relying on the Safe Theorem, hyperstatic equilibrium is also taken into account.

\section{THE FINITE ELEMENT METHOD}

We now quickly survey the Finite Element Method, particularly focusing on the analysis of masonry structures. Excellent textbooks [Bathe 2006] cover the method in detail, and we refer to other surveys [Müller et al. 2008; Sifakis and Barbic 2012] for more extensive discussion of the application of the FEM to computer graphics simulations. While there are numerous FEM formulations with different assumptions, we first introduce the simplest, most naïve version of the algorithm with a linear material model and continuum contact constraints. Although this may seem like a simplistic model, it is the version that is most commonly implemented in commercial software and widely employed by practitioners [Autodesk 2014; Computers and Structures, Inc. 2014; Dassault Système 2014]. This is the case even for masonry analysis, although it has been demonstrated that the results are unreliable in some cases (e.g., Block et al. [2006]). Later, we also consider different variations that improve on this method.

Linear Elastic Material. The linear FEM treats structures as a finite set of elements of finite size. Each element is defined by a set of nodes, and it is assumed that a linear stress-strain relationship holds across the element, encoded as a per-element stiffness matrix. The stiffness matrix depends on the physical behavior of the material, and for homogeneous and isotropic elastic materials it is usually characterized by the Lamé parameters, or Young's modulus and Poisson's ratio. A global stiffness matrix, K, can be constructed for the entire physical system by assembling the element stiffness matrices. $\mathbf{K}$ relates nodal displacements $\left(\mathbf{x}_{\mathrm{aft}}-\mathbf{x}_{\mathrm{bfr}}\right)$ to internal forces at element nodes $\left(\mathbf{f}_{\mathrm{in}}^{n}\right)$, where $\mathbf{x}_{\mathrm{bfr}}$ and $\mathbf{x}_{\mathrm{aft}}$ refer, respectively, to nodal positions before and after the structure undergoes deformation.

$$
\mathbf{f}_{\mathrm{in}}^{n}=\mathbf{K}\left(\mathbf{x}_{\mathrm{aft}}-\mathbf{x}_{\mathrm{bfr}}\right)
$$

For most physical materials, the simplified linear stress-strain relationship and multilinear strain measure is a good approximation for small displacements.

Contact Constraints. Contact constraints limit the space of allowable nodal displacements. The simplest FEM models the structure as a continuum (i.e., a monolith)—displacements $\left(\mathbf{x}_{\mathrm{aft}}-\mathbf{x}_{\mathrm{bfr}}\right)$ across the structure are continuous and fracture is disallowed. This condition is expressed as a constraint that overlapping nodes on adjacent elements have identical position:

$$
x_{\mathrm{aft}}^{j}=x_{\mathrm{aft}}^{k} \quad \forall j, k \text { s.t. }\left(x_{\mathrm{bfr}}^{j}=x_{\mathrm{bfr}}^{k}\right) .
$$



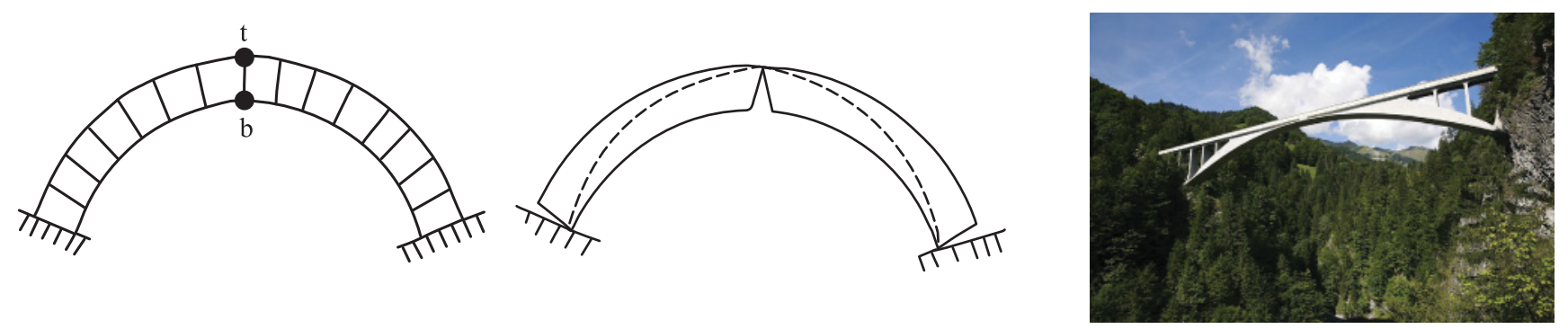

Fig. 5. A classic example of a structure that is stable, despite its blocks not resting flush against each other, is the three-hinged arch. Begin with a stable circular arch (left) and pull the base apart (center). The two halves of the arch hinge inward, with the top two blocks remaining in contact only along their top boundary $t$. Thrust line analysis demonstrates that the hinged configuration is still stable, since a thrust line exists embedded within the volume of the arch. Many real-world bridges, such as the Salginatobel Bridge in the Swiss Alps (right), stand by this principle.

Over the entire structure this constraint can be expressed as a sparse linear system of equalities:

$$
\mathbf{A}_{\Omega}\left(\mathbf{x}_{\mathrm{aft}}-\mathbf{x}_{\mathrm{bfr}}\right)=0,
$$

where $\mathbf{A}_{\Omega}$ is a sparse matrix of coefficients.

This constraint models the entire structure as a continuum. In traditional FEM, the element boundaries are artificial boundaries inside a continuum object that have both compressive and tensile capacity. The FEM computes the deformation of the object, which causes internal stress distributed throughout the elements. When a node is subject to stress that exceeds its capacity, the object undergoes fracture or material failure at this location.

As mentioned earlier, while the compressive strength of stones is extremely high and practically infinite, compared to the average working stress, the same is not true for tensile strength, particularly for masonry structure built of weakly connected blocks. Mortar between blocks appears to connect the structure, but in practice it is very weak and provides close to zero tensile capacity. Tensile stress at the interfaces of blocks has thus been interpreted as an indication that the masonry structure is not safe (e.g., Brune and Perucchio [2012]).

Here lies the important difference between masonry structures and other continuum structures modeled in traditional FEM. Block interfaces in masonry are physical boundaries that have practically zero tensile capacity. Tensile stresses at the interface mean that the adjacent elements or blocks will separate from each other. However, in case of masonry, this does not automatically mean that the structure will collapse. Blocks can hinge without completely separating from each other, and in fact, some stable configurations of structures involve such hinging.

The arch in Figure 5 is an example of a stable hinged structure. The three-hinged arch in the center is formed by taking a stable circular arch and pulling the bases apart. At the top part, $t$, of the crown interface, the blocks are pushing against each other, so the adjacency constraint applies. On the other hand, as the base is pulled apart, at the bottom part of the same interface, $b$, and at the outer parts of the base interfaces, the blocks separate away, forming a hinge. The three-hinged arch is a well-known stable configuration [Heyman 1996], and many real-world arch bridges stand by this principle. Notice that in the final stable configuration, there is no tensile stress at the opening of the hinges. The blocks simply separate from each other at these locations. In order for the FEM to find such configurations, contact constraints must be carefully specified to express the physical reality, allowing separation between blocks and disallowing interpenetration. Naïve continuum FEM does not do this.
Many different strategies have been developed to address this issue. For example, Brasile et al. [2007] and Lourenço [1998] use anisotropic continuum models for separate brick and mortar layers. Dolbow and Belytschko [1999] developed the eXtended Finite Element Method (XFEM) to model fractures and discontinuities by enriching the solution space with discontinuous functions, and Cundall et al. [1971] introduced the Discrete Element Method (DEM), which simulates discrete particles and their contact through time. These methods are computationally expensive and require tuning parameters [DeJong 2009]. Perhaps for these reasons, they are rarely used by practitioners.

In graphics simulations, it is a well-known technique to add inequality constraints to the contact constraints to express unilateral contacts [Baraff 1993]. Do correct contact constraints reconcile the differences between equilibrium methods and FEM when analyzing masonry stability? That is, can the FEM with correct contact constraints predict equilibrium of a masonry structure? As we will see in Section 5, the answer is "not quite." But before we examine a failure case in detail, we will finish describing the FEM solution and make some remarks about how the FEM equations relate to those of the block equilibrium method.

Deformation Solution. Once the material properties and contact constraints are appropriately assigned, the FEM solves for the deformation of the structure $\left(\mathbf{x}_{\mathrm{aft}}\right)$ at static equilibrium. The principle of virtual work states that the equilibrium configuration minimizes the sum of elastic strain energy stored in the structure, $\frac{1}{2}\left(\mathbf{x}_{\mathrm{aft}}-\mathbf{x}_{\mathrm{bfr}}\right)^{\top} \mathbf{K}\left(\mathbf{x}_{\mathrm{aft}}-\mathbf{x}_{\mathrm{bfr}}\right)$, the gravitational potential energy and the work done by external forces, $\mathbf{f}_{\mathrm{w}}^{n \top}\left(\mathbf{x}_{\mathrm{aft}}-\mathbf{x}_{\mathrm{bfr}}\right)$ :

$U\left(\mathbf{x}_{\mathrm{bfr}}, \mathbf{x}_{\mathrm{aft}}\right)=\frac{1}{2}\left(\mathbf{x}_{\mathrm{aft}}-\mathbf{x}_{\mathrm{bfr}}\right)^{\top} \mathbf{K}\left(\mathbf{x}_{\mathrm{aft}}-\mathbf{x}_{\mathrm{bfr}}\right)+\mathbf{f}_{\mathrm{w}}^{n \top}\left(\mathbf{x}_{\mathrm{aft}}-\mathbf{x}_{\mathrm{bfr}}\right)$,

where $\mathbf{f}_{\mathrm{w}}^{n}$ is the force applied by self-weight and external loads.

The FEM is then a quadratic program that given an input undeformed geometry $\left(\tilde{\mathbf{x}}_{\mathrm{bfr}}\right)$ solves for the deformed geometry $\left(\mathbf{x}_{\mathrm{aft}}\right)$ :

$$
\mathbf{x}_{\mathrm{aft}}^{*}=\underset{\mathbf{x}_{\mathrm{aft}}}{\operatorname{argmin}} U\left(\mathbf{x}_{\mathrm{aft}}, \tilde{\mathbf{x}}_{\mathrm{bfr}}\right)
$$

s.t. Contact forces at block interfaces are compressive

Inequality constraints express the compression-only constraint on all adjacent nodes at the interfaces:

$$
\left(\mathbf{x}_{\mathrm{aft}}^{j}-\mathbf{x}_{\mathrm{aft}}^{k}\right) N\left(\mathbf{x}_{j k}\right) \leq 0 \quad \forall j, k \text { s.t. }\left(\mathbf{x}_{\mathrm{bfr}}^{j}=\mathbf{x}_{\mathrm{bfr}}^{k}\right),
$$

where $N\left(x_{j k}\right)$ is the normal direction of the interface where nodes $x_{j}$ and $x_{k}$ meet, pointing away from $x_{j}$. If it is known a priori which nodes are in compression and which are in tension, the compression-only constraint can be expressed instead as a linear 


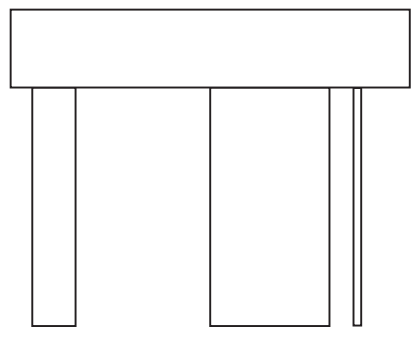

(a) Un-deformed Geometry

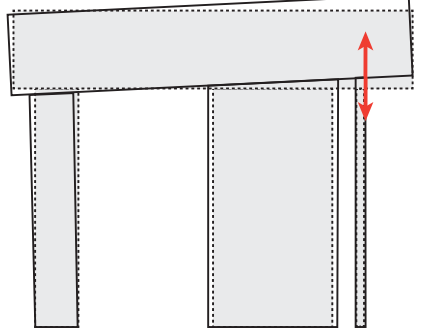

(b) Naïve FEM $\left(0.0^{\circ}\right)$

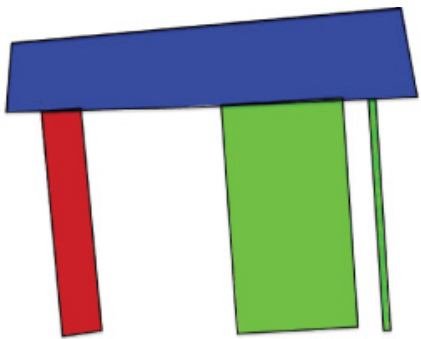

(c) FEM w Inequality Constraints $\left(3.0^{\circ}\right)$

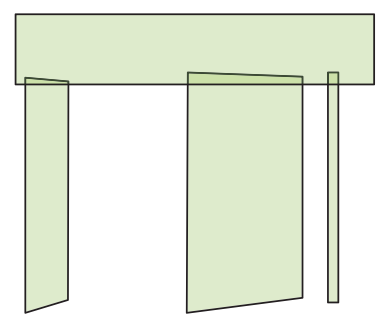

(d) Inverse FEM $\left(19.1^{\circ}\right)$

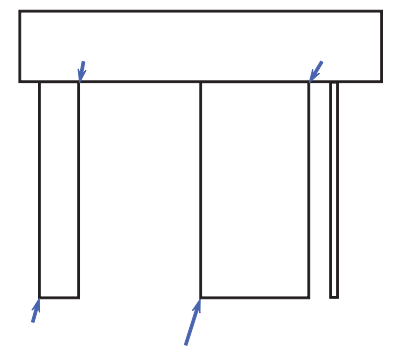

(e) Block Equilibrium $\left(19.1^{\circ}\right)$

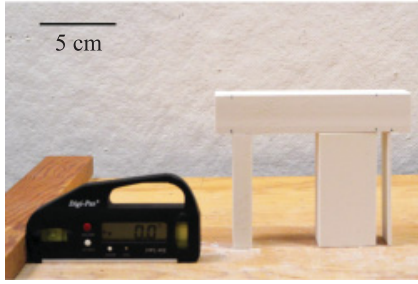

(f) Physical Model $\left(0.0^{\circ}\right)$

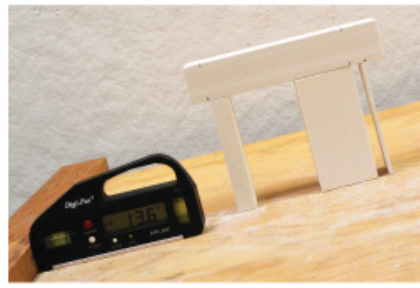

(g) Physical Model $\left(13.6^{\circ}\right)$

Fig. 6. The three-legged $\pi$ geometry is a simple model that illustrates the problem with the forward FEM. A horizontal block rests on three supports of equal height but varying width. (a) The undeformed geometry of the three-legged $\pi$. (b) The deformed, settled state of the $\pi$ model using finite elements with naïve (continuum) contact constraints between elements. The method predicts tension at the indicated location on the right block. (c) An Abaqus simulation of the tilted $\pi$ model with inequality contact constraints still incorrectly predicts toppling of the structure at $3.0^{\circ}$. (d) Inverse FEM (Section 6) and (e) block equilibrium method (Section 3) both predict that the tilted $\pi$ is stable up to $19.1^{\circ}$. The solved-for rest shape of the blocks and one possible set of contact forces certifying equilibrium of the structure are shown for the two methods, respectively. (f) We performed an experimental tilt test of the three-legged $\pi$. (g) The $\pi$ stands even when tilted to $13.6^{\circ}$, well beyond the point that failure was predicted by forward FEM simulation.

constraint on the active set by adjusting $\tilde{\mathbf{A}}_{\Omega}$, the contact constraint in Equation (7), to apply only to adjacent vertices in compression. In either case, the blocks are allowed to hinge and prevented from interpenetrating.

Note that the output of the previous optimization $\left(\mathbf{x}_{\mathrm{aft}}^{*}\right)$ is a deformed structure-the input geometry $\left(\tilde{\mathbf{x}}_{\text {bfr }}\right)$ is not preserved but settles into a minimum energy state. This does not answer quite the same question as the inverse problem: given the geometry of the masonry "as it lies (already settled or deformed)," does there exist a physically plausible undeformed state for which the current configuration is stable? Since masonry is stiff and displacements are very small, it seems reasonable to assume that this difference is negligible. We will return to this point in Section 6.

Comparison to the Equilibrium Method. The equations of the FEM presented previously turn out to be quite similar to that of the equilibrium methods in Section 3. This is clearer if we look at the dual formulation of the FEM. The Lagrangian form of the FEM Equation (9) is

$$
L\left(\mathbf{x}_{\mathrm{aft}}, \lambda\right)=U\left(\mathbf{x}_{\mathrm{aft}}\right)+\lambda^{\top} \tilde{\mathbf{A}}_{\Omega}\left(\mathbf{x}_{\mathrm{aft}}-\tilde{\mathbf{x}}_{\mathrm{bfr}}\right),
$$

where $U$ is the potential energy as a function of the deformed geometry $\left(\mathbf{x}_{\mathrm{aft}}\right), \tilde{\mathbf{A}}_{\Omega}\left(\mathbf{x}_{\mathrm{aft}}-\mathbf{x}_{\mathrm{bfr}}\right)$ is the contact constraint, and $\lambda$ is the vector of Lagrange multipliers. Taking the derivative of the Lagrangian with respect to $\mathbf{x}_{\text {aft }}$, we get

$$
\frac{d L}{d \mathbf{x}_{\mathrm{aft}}}=\tilde{\mathbf{K}}\left(\mathbf{x}_{\mathrm{aft}}-\tilde{\mathbf{x}}_{\mathrm{bfr}}\right)+\tilde{\mathbf{f}}_{\mathrm{w}}^{n}+\tilde{\mathbf{A}}_{\Omega}^{\top} \lambda .
$$

At $\mathbf{x}_{\mathrm{aff}}^{*}$, the minimum potential energy state, the derivative is equal to zero:

$$
\tilde{\mathbf{K}}\left(\mathbf{x}_{\mathrm{aft}}^{*}-\tilde{\mathbf{x}}_{\mathrm{bfr}}\right)+\tilde{\mathbf{f}}_{\mathrm{w}}^{n}+\tilde{\mathbf{A}}_{\Omega}^{\top} \lambda=\mathbf{f}_{\mathrm{in}}^{n}+\tilde{\mathbf{f}}_{\mathrm{w}}^{n}+\mathbf{f}_{\mathrm{ct}}^{n}=0,
$$

where $\tilde{\mathbf{K}}\left(\mathbf{x}_{\mathrm{aft}}^{*}-\tilde{\mathbf{x}}_{\mathrm{bfr}}\right)=\mathbf{f}_{\mathrm{in}}^{n}$ is the elastic force, $\tilde{\mathbf{f}}_{\mathrm{w}}^{n}$ is the force from self-weight and external loads, and $\tilde{\mathbf{A}}_{\Omega}^{\top} \lambda=\mathbf{f}_{\mathrm{ct}}^{n}$ is the contact force induced by the contact constraints, all defined at the element nodes. Equation (13), the condition that the derivative of the Lagrangian is zero at the solution, turns out to be the condition that the net force per element node equals zero. Compare this to the equilibrium Equations (1) and (2) in the block equilibrium method. Equilibrium methods state that the net force and net torque are zero per block. Also, whereas the compression-only constraint is directly specified as an essential part of the equilibrium method, in the FEM it is applied to the contact constraints in an implicit manner (by specifying the active set where the contact constraints apply).

\section{THE PROBLEM WITH FORWARD FEM}

Despite the similarity of the FEM formulation to the equilibrium methods, the FEM, even with correct one-sided contact constraints, still fails to predict a no-tension equilibrium of certain structures. Consider the three-legged $\pi$ model in Figure 6, which consists of a long horizontal block balanced on top of three vertical columns of different thicknesses. The structure is clearly stable; the center of mass of the top block falls inside the support region of the columns, defined by the convex hull of the parts of the structure 
Table III. Comparison of Naïve FEM, Inverse FEM, and Equilibrium Methods

\begin{tabular}{c|ccc}
\hline Method & Naïve FEM & Inverse FEM & Equilibrium \\
\hline Solution variable & Deformed geometry $\left(\mathbf{x}_{\mathrm{aft}}\right)$ & Undeformed geometry $\left(\mathbf{x}_{\mathrm{bfr}}\right)$ & Contact forces at interfaces $\left(\mathbf{f}_{\mathrm{ct}}^{i}\right)$ \\
Constraints & Nodal displacements & Lagrangian derivative & Sum force and torque \\
Objective function & Minimum potential energy & None / User defined & None / User defined \\
Equilibrium condition & Sum force per element node & Sum force and torque per block \\
\hline
\end{tabular}

touching the ground. Let us examine closely what happens to the structure according to the FEM described in the previous section. Due to the force of gravity and the weight of the top block pushing against them, the three columns deform and shorten. Since the leftmost column is thinner and carries more of the weight, it shortens slightly more than the middle column. As a result, the top block tilts leftward leaning against the left and the middle column. If the structure is treated as a monolith with the naïve contact constraint in Equation (6), artificial tensile stresses appear at the interface of the rightmost column and the top block (Figure 6, top left). If we follow the practice of interpreting tension at block interfaces as an indicator of structural failure, we would make an incorrect conclusion that the structure cannot stand.

Replacing the continuum contact constraints by inequality contact constraints allows hinging at the block interfaces. However, this still does not guarantee a correct stability prediction. Consider what happens if we start tilting the ground counterclockwise (i.e., the structure is standing on a slope). According to the inequalityconstrained FEM, soon, a hinge starts to form between the leftmost column and the top block. The top block tilts leftward, lifting up from the rightmost block. Very soon, the center of mass of the rightmost block falls outside its support region, and the column topples over, causing the structure to fail. Indeed, when we run the experiment on Abaqus with discontinuous boundaries at the block interfaces, it failed to find an equilibrium solution for the structure at $3.0^{\circ}$. However, when we analyze the structure's stability using the block equilibrium method and perform a physical experiment of the same model, we see that the structure can tilt well beyond $3.0^{\circ}$ (see Figure 6, bottom). What is causing the discrepancy?

Naturally, it is possible to improve the FEM further by adding more sophisticated features. For instance, we can model nonlinear stress-strain relationships or use a nonlinear strain measure. In fact, numerous variations of the FEM allow such modifications at the expense of increasing complexity and computational cost [Lourenço et al. 1998; Cundall 1971; Dolbow and Belytschko 1999]. However, it is unclear exactly which of the assumptions mentioned in the introduction are responsible for the FEM's error and hence which modifications are worth their cost when it comes to answering the question, "will the structure stand?"

In the next section, we turn to the key advantage of equilibrium methods and apply it to FEM to make it more suitable for masonry analysis. We show that with only a simple modification to the original FEM formulation and without adding more complexity, we can obtain the same predictions as equilibrium methods.

\section{INVERSE FINITE ELEMENT METHOD}

One of the main advantages of equilibrium methods is that they treat the blocks as rigid bodies. Since the blocks do not displace, handling of the contact constraints is simple: we need not worry about contacts forming or breaking over time (due to, e.g., hinging). Since the material properties are already built into the model, equilibrium methods also avoid the need for specifying other material parameters. This greatly simplifies the problem formulation.

We can avoid the problem of contact resolution in the FEM if we know the final geometry. So, instead of solving for the deformation of the input geometry, consider the following modification: let the input be the deformed state. Now, given the inputdeformed geometry, which is the final minimum energy state $\left(\tilde{\mathbf{x}}_{\mathrm{aft}}^{*}\right)$, solve for the original undeformed geometry $\left(\mathbf{x}_{\mathrm{bfr}}\right)$. Hence, the original FEM Equation (9) becomes

$$
\tilde{\mathbf{x}}_{\mathrm{aft}}=\underset{\mathbf{x}_{\mathrm{aft}}}{\operatorname{argmin}} U\left(\mathbf{x}_{\mathrm{aft}}, \mathbf{x}_{\mathrm{bfr}}\right)
$$

s.t. Contact forces at block interfaces are compressive,

where $\mathbf{x}_{\mathrm{bfr}}$ is the unknown, and $U$ is the potential energy of the structure from the original FEM formulation, Equation (8). Since the final geometry (and hence the normal direction of each interface, $N\left(\mathbf{x}_{j k}\right)$, in the compression-only constraint) is known, we can express it as a linear inequality constraint:

$$
\tilde{\mathbf{A}}_{\mathrm{N}}\left(\mathbf{x}_{\mathrm{aft}}-\mathbf{x}_{\mathrm{bfr}}\right) \leq 0,
$$

where $\tilde{\mathbf{A}}_{\mathrm{N}}$ is a sparse matrix of coefficients encoding the normal direction and adjacency of element nodes.

To solve for $\mathbf{x}_{\mathrm{bfr}}$, take the Lagrangian form of the new FEM Equation (14):

$$
L\left(\mathbf{x}_{\mathrm{aft}}, \mathbf{x}_{\mathrm{bfr}}, \lambda\right)=U\left(\mathbf{x}_{\mathrm{aft}}, \mathbf{x}_{\mathrm{bfr}}\right)+\lambda^{\top} \tilde{\mathbf{A}}_{\mathrm{N}}\left(\mathbf{x}_{\mathrm{aft}}-\mathbf{x}_{\mathrm{bfr}}\right) .
$$

Since the minimum solution is given as the input $\left(\tilde{\mathbf{x}}_{\mathrm{aft}}\right)$, we can write

$$
\begin{aligned}
\left.\frac{d L}{d \mathbf{x}_{\mathrm{aft}}}\right|_{\tilde{\mathbf{x}}_{\mathrm{aft}}} & =\tilde{\mathbf{K}}\left(\tilde{\mathbf{x}}_{\mathrm{aft}}-\mathbf{x}_{\mathrm{bfr}}\right)+\tilde{\mathbf{f}}_{\mathrm{w}}^{n}+\tilde{\mathbf{A}}_{\mathrm{N}}^{\top} \lambda \\
& =\mathbf{f}_{\mathrm{in}}^{n}+\tilde{\mathbf{f}}_{\mathrm{w}}^{n}+\mathbf{f}_{\mathrm{ct}}^{n} \\
& =0
\end{aligned}
$$

where the unknowns are the undeformed geometry $\left(\mathbf{x}_{\mathrm{bfr}}\right)$ and the Lagrangian multiplier $(\lambda)$. The stiffness matrix depends nonlinearly on the nodal rest positions $\mathbf{x}_{\mathrm{bfr}}$, but since displacements will be small, its dependence on the rest positions is negligible (see appendix for derivation). Similar to Equation (13), which we get from the Lagrangian of the forward FEM, Equation (17) is an equilibrium condition that states that for each node, the sum of the forces equals zero. The difference is that in the inverse FEM, the final geometry and hence the solution to the optimization, $\mathbf{x}_{\mathrm{aft}}^{*}$, is known, whereas in the forward FEM, only the undeformed geometry $\left(\mathbf{x}_{\text {bfr }}\right)$ is known. Fixing the final geometry resolves the contact problem naturally. The nodes that are adjacent in the input geometry are the ones that are also in contact after the deformation.

Similar to the equilibrium methods, the inverse FEM searches for one of possibly many solutions $\left(\mathbf{x}_{\text {bfr }}\right.$ and $\lambda$ ) that satisfy the equilibrium condition in Equation (17). Likewise, in practice a userdefined objective function $(\mathbf{H})$ is added to obtain a unique solution. For instance, $\mathbf{H}$ can be set to minimize the amount of deformation, $\left(\tilde{\mathbf{x}}_{\mathrm{aft}}-\mathbf{x}_{\mathrm{bfr}}\right)$, or the internal force induced by the deformation, $\tilde{\mathbf{K}}\left(\tilde{\mathbf{x}}_{\mathrm{aft}}-\right.$ $\left.\mathbf{x}_{\mathrm{bfr}}\right)$. Finally, the solution is obtained by solving the optimization problem:

$$
\begin{aligned}
{\left[\mathbf{x}_{\mathrm{bfr}}^{*}, \lambda^{*}\right]=\underset{\mathbf{x}_{\mathrm{bfr}}, \lambda}{\operatorname{argmin}} \mathbf{H}\left(\mathbf{x}_{\mathrm{bfr}}, \lambda\right) } \\
\text { s.t. } \quad \tilde{\mathbf{K}}\left(\tilde{\mathbf{x}}_{\mathrm{aft}}-\mathbf{x}_{\mathrm{bfr}}\right)+\tilde{\mathbf{f}}_{\mathrm{w}}^{n}+\tilde{\mathbf{A}}_{\mathrm{N}}^{\top} \lambda .
\end{aligned}
$$


So both the inverse FEM and the equilibrium approach keep the input geometry as the final geometry of the structure. Both constrain the contact forces to act in compression only, and both solve for a possible solution state that satisfies some equilibrium condition. In case of the inverse FEM, the equilibrium condition is in terms of net force per element node, and in case of the equilibrium methods, it is net force and torque per block. Next, we prove that, in fact, these equilibrium conditions are equivalent.

\section{Equivalence of the Inverse FEM and Equilibrium Methods}

I. Forces induced by the displacements solved for using the inverse FEM also satisfy the constraints of the block equilibrium method. In other words, every solution of the inverse FEM problem corresponds to a set of forces at the block interfaces that are solutions of the block equilibrium method.

The equilibrium condition of inverse FEM, Equation (17), states that the sum of the forces acting on each element node is zero. It easily follows that the sum of the forces acting on each block $(b)$ is also zero:

$$
\sum_{i \in b}\left(\mathbf{f}_{\mathrm{in}}^{i}+\mathbf{f}_{\mathrm{w}}^{i}+\mathbf{f}_{\mathrm{ct}}^{i}\right)=0 \quad \forall b .
$$

Since the elastic energy of each block is invariant under uniform translation of the block's nodes, by Noether's theorem, the gradient of elastic energy has no component of rigid translation and

$$
\sum_{i \in b} \mathbf{f}_{\text {in }}^{i}=0 \quad \forall b .
$$

That is, the sum of internal forces per block is zero. Subtracting Equation (20) from Equation (19), it follows that

$$
\sum_{i \in b}\left(\mathbf{f}_{\mathrm{w}}^{\mathrm{i}}+\mathbf{f}_{\mathrm{ct}}^{i}\right)=0 \quad \forall b .
$$

That is, the sum of contact forces, external forces, and selfweight is zero. This is the exactly the per-block force balance in Equation (1). An identical argument holds for infinitesimal rotation of the blocks and torque balance at the center of mass (see appendix for full proof).

II. For a given input geometry, if the block equilibrium method finds a set of forces that puts the geometry in static equilibrium, then there exists an undeformed (rest) configuration of the input geometry that is in static equilibrium when deformed to the input positions. In other words, every set of forces at the block interfaces that are solutions of the block equilibrium method correspond to some rest configuration that certifies stability of the input geometry as judged by the inverse FEM.

Let $\mathbf{f}_{\mathrm{in}}^{i}=-\mathbf{f}_{\mathrm{ct}}^{i}-\mathbf{f}_{\mathrm{w}}^{i}$. By definition, these forces satisfy the inverse FEM equilibrium equation:

$$
\mathbf{f}_{\mathrm{in}}^{i}+\mathbf{f}_{\mathrm{w}}^{i}+\mathbf{f}_{\mathrm{ct}}^{i}=0 \quad \forall i .
$$

It remains only to show that there exists an undeformed configuration that generates these elastic forces-a deformation $\mathbf{u}=\tilde{\mathbf{x}}_{\mathrm{aft}}-\mathbf{x}_{\mathrm{bfr}}$ for which $\mathbf{f}_{\text {in }}=\mathbf{K u}$. It suffices to show this on each block b separately. The Fredholm alternative states that exactly one of the following cases holds:

(a) $\mathbf{f}_{\text {in }}^{b}=\mathbf{K}^{b} x$ has a solution. In this case, $x$ is the desired undeformed positions of the nodes of $b$.

(b) $\left(\mathbf{K}^{b}\right)^{\top} y=0$ has a solution $y$ with $y^{\top} \mathbf{f}_{\text {in }}^{\mathrm{b}} \neq 0$. But $\mathbf{K}^{b}$ is symmetric, and the only vectors in its kernel are the infinitesimal rigid translations and rotations of the block. Therefore, $y$ can be decomposed into pure translation (t) and infinitesimal rotation (r) components.

$$
\begin{aligned}
y^{\top} \mathbf{f}_{\mathrm{in}}^{b} & =(\mathbf{t}+\mathbf{r})^{\top} \mathbf{f}_{\mathrm{in}}^{b} \\
& =\mathbf{t} \cdot \mathbf{f}_{\mathrm{in}}^{b}+\left[\omega \times\left(\mathrm{x}_{\mathrm{aft}}^{b}-\mathbf{c}_{\mathrm{aft}}^{b}\right)\right] \cdot \mathbf{f}_{\mathrm{in}}^{b}=0,
\end{aligned}
$$

where $\mathbf{c}_{\mathrm{aft}}^{b}$ is the block's center of mass, and $\omega$ is the rotation vector of $\mathbf{r}$ and is understood to act per node on the torque arm, $\mathbf{x}_{\mathrm{aft}}^{\mathrm{i}}-\mathbf{c}_{\mathrm{aft}}^{\mathrm{i}}$. The last equality $(=0)$ follows since

$$
\left[\omega \times\left(\mathbf{x}_{\mathrm{aft}}^{b}-\mathbf{c}_{\mathrm{aft}}^{b}\right)\right] \cdot \mathbf{f}_{\mathrm{in}}^{b}=\left[\left(\mathbf{x}_{\mathrm{aft}}^{b}-\mathbf{c}_{\mathrm{aft}}^{b}\right) \times \mathbf{f}_{\mathrm{in}}^{b}\right] \cdot \omega=0,
$$

and by the block equilibrium method's constraints, $\mathbf{f}_{\text {in }}^{b}$ does not have a force or torque component. Equation (23) contradicts (b). Therefore, (a) is true and $\mathbf{f}_{\text {in }}^{b}=\mathbf{K}^{b} x$ has a solution.

We have shown that the inverse FEM obtains results equivalent to those of the block equilibrium method and vice versa. Both approaches to stability analysis can be viewed as equivalent, dual methods for getting the same answer to the same problem, with the FEM formulating the problem in terms of displacement variables, and equilibrium methods in terms of force variables. By making the final geometry a known input, both formulations avoid the difficulty of specifying the contact constraints. The block equilibrium method has the additional simplicity of expressing the compression-only constraint directly in terms of forces. Inverse FEM expresses the same constraint, but indirectly as a linear inequality constraint in terms of displacements (see Equation (15)).

\section{EXPERIMENTAL RESULTS AND VALIDATION}

For several different example structures, both didactic and complex, we compared the stability of the structure as predicted by the following methods:

-naïve FEM using continuum, linear elastic finite elements, the most widely used computational analysis used by practitioners. (We expect it to give overconservative results far from the analytical or the physical experiment results);

-FEM with inequality contact constraints, still using forward simulation. This one-sided handling of contact constraints improves upon the previous model, but small deformations in the elements can translate to nonlinear changes in the geometry and topology of the block network that lead to inaccurate results;

- the block equilibrium method;

-inverse FEM (as we saw in the previous section, this method ought to give identical results as the block equilibrium method);

- exact analysis (for the simple examples where the analytical solution is tractable);

— for five of the models, physical experiment.

To most effectively evaluate the predictions of these methods, we subjected each structure to a tilt test: we continuously tilted the ground plane on which the structure rests and computed the critical angle at which the structure topples. In the simulations, this corresponds to increasing the horizontal component of the gravitational acceleration. This tilt analysis is commonly used as an initial assessment of the lateral stability of structures [Zessin 2012].

We use the commercial software package Abaqus/Standard [Dassault Système 2014] for the forward FEM implementations. For all examples, we used linear elastic material with Young's modulus $1 \mathrm{MPa}$, Poisson's ratio 0.2, and uniform density. Detailed settings for Abaqus parameters are attached in the appendix, as well as displacement scaling factors used to aid visualization. 
Table IV. Tilt Test

\begin{tabular}{|c|c|c|c|c|c|c|}
\hline \multirow{2}{*}{$\begin{array}{c}\text { Model } \\
\text { (\# of blocks) }\end{array}$} & \multicolumn{6}{|c|}{ Critical Tilt Angle $\left(^{\circ}\right)$} \\
\hline & Naïve FEM & FEM w Inequality & Inverse FEM & Equilibrium & Analytic & Experiment \\
\hline Cube (1) & 18.4 & 44.8 & 45.0 & 45.0 & 45.0 & $41.0 \pm 0.3$ \\
\hline Arch, infeasible (36) & infeasible & infeasible & infeasible & infeasible & infeasible* & - \\
\hline Arch, thin (36) & infeasible & infeasible & 0.1 & 0.1 & $0.1^{*}$ & - \\
\hline Arch, thick (36) & infeasible & 7.2 & 8.2 & 8.2 & $8.2^{*}$ & $4.7 \pm 0.2$ \\
\hline Gothic $\operatorname{arch}(7)$ & 0.1 & 16.8 & 17.4 & 17.4 & - & $15.7 \pm 0.2$ \\
\hline 3-legged $\pi(4)$ & infeasible & 3.0 & 19.1 & 19.1 & - & $14.3 \pm 0.2$ \\
\hline Flying Buttress (22) & infeasible & 0.0 & 5.7 & 5.7 & - & $4.4 \pm 0.7$ \\
\hline Aqueduct (490) & infeasible & infeasible & 6.5 & 6.5 & - & - \\
\hline
\end{tabular}

*Analytical angles for circular arch [Ochsendorf 2002].

For the physical experiments, we created small-scale models by assembling $3 \mathrm{D}$ printed blocks. The blocks were printed using a ZCorp 400 Series powder printer with clear binder. After printing, blocks were infiltrated with 3D Systems ColorBond, also commonly referred to as Z-Bond 90, in order to reduce material deterioration from multiple tests. The material density was measured to be $1.5 \mathrm{~g} / \mathrm{cm}^{3}$ with a friction angle of $43^{\circ}$. We used a tilt table consisting of a stiff platform attached to a hinge along one edge and a string along the opposite edge. To minimize dynamic effects, the table was tilted by pulling the string at a very low speed. The models were tilted to collapse three times. All of the experiments were filmed with a high-speed video camera, and the angle of tilt was monitored with a digital protractor.

For each method, we determined the failure of a structure by the following criteria. The inverse FEM and equilibrium methods predict failure when a feasible solution that satisfies their respective equilibrium conditions do not exist. For naïve FEM, we interpreted tensile forces at the block interfaces as failure, since we are assuming they have zero tensile capacity. FEM with inequality contact constraints cannot predict tensile forces at block interfaces since blocks are allowed to separate from each other. Instead, we measured the critical tilt angle when Abaqus failed to converge to an equilibrium solution. This can happen, for example, when the displacements of the blocks become too large (e.g., when the blocks topple). Admittedly, the Abaqus results can be more or less accurate depending on numerous variables, such as the exact way blocks are discretized into mesh elements, the maximum number of iteration attempts allowed for the solver, or the numerical precision/tolerance settings. Finding the right parameters that work for each model is not straightforward and is a major source of difficulty in correctly analyzing masonry stability using elastic analysis. We have experimented with changing the parameters (see appendix on Abaqus settings for detail) and show the least conservative critical angle.

Table IV shows the result of the experiments. The models we tested are, in order of increasing complexity, as follows:

Simple Cube. A lone cube should hinge and topple when tilted past the point where its center of mass lies over its support, at $45^{\circ}$. With the exception of continuum $\mathrm{FEM}^{1}$, all methods predict the correct critical angle for this trivial example.

Circular Arches. The stability of a circular arch can be characterized by the dimensionless thickness-to-radius ratio $t / r$ : any arch with $t / r<0.1075$ is unstable, and the arch becomes increasingly stable as $t / r$ increases. Analytic solutions for the critical angle are

\footnotetext{
${ }^{1} \mathrm{~A}$ cube is a single continuum block. For this example only, to distinguish between naive FEM and the formulation with inequality constraints, the naive FEM models the cube and the ground as a single continuum (i.e., the block is glued to the ground). Failure is predicted when tension first appears at the interface between the ground and the block.
}

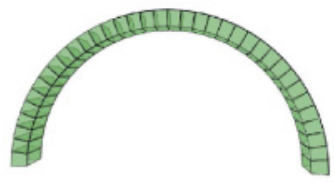

(a) Inverse FEM (thin, $0.0^{\circ}$ )

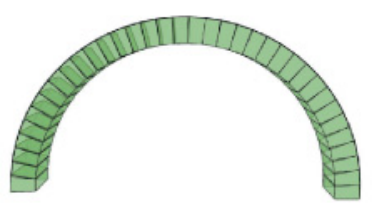

(c) Inverse FEM (thick, $8.2^{\circ}$ )

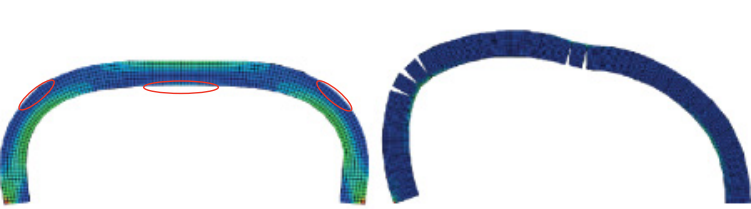

(e) Naïve FEM (thick, $0.0^{\circ}$ )

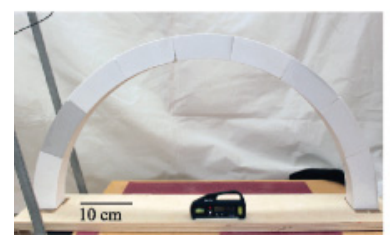

(g) Physical Model $\left(0.0^{\circ}\right)$

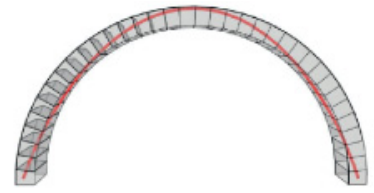

(b) Equilibrium (thin, $0.0^{\circ}$ )

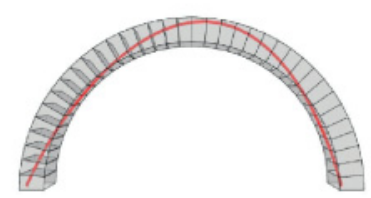

(d) Equilibrium (thick, 8.2 $2^{\circ}$
Fig. 7. Results of the tilt-test applied to circular arches. (a) Inverse FEM and (b) the block equilibrium method both correctly predict that an arch with thickness-to-radius ratio 0.1075 , the thinnest possible stable circular arch, is stable. For a thicker $\operatorname{arch}(t / r=0.150)$, both the (c) inverse FEM and (d) block equilibrium method correctly predict that the arch is stable up to a tilt angle of $8.2^{\circ}$. (e) The naïve continuum FEM predicts tension at block interfaces (circled) even at the rest position $\left(0.0^{\circ}\right)$. (f) With corrected contact constraints that allow hinging between blocks, forward FEM predicts failure at a more accurate, but still overly conservative, angle of $7.2^{\circ}$. (g, h) In a physical tilt test, the model fails at $4.7^{\circ}$, likely due to imperfections in the fabrication process of the blocks, corresponding to an effectiveness thickness of $87 \%$.

available [Ochsendorf 2002]. Even at the horizontal rest position, the naïve continuum FEM predicts failure due to finding tension at the interfaces. Note that this does not mean that the structure will fail. Rather, it means that hinges are trying to form between blocks but they are prevented from doing so because of incorrect contact 


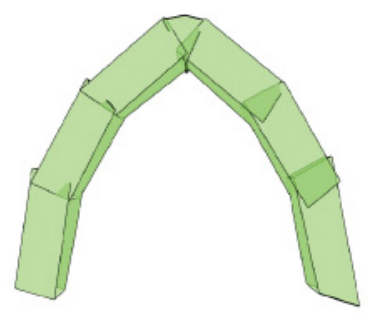

(a) Inverse FEM $\left(17.4^{\circ}\right)$

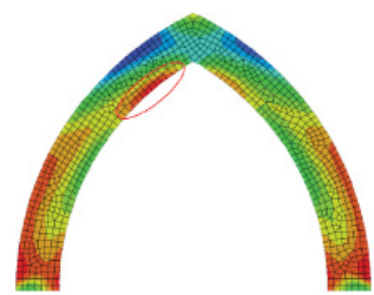

(c) Naïve FEM $\left(0.1^{\circ}\right)$

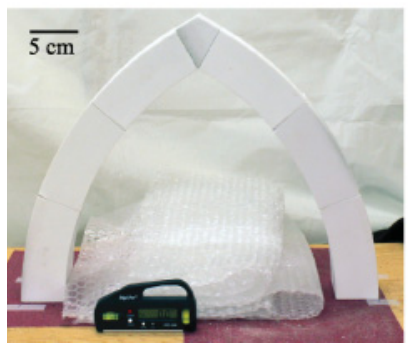

(e) Physical Model $\left(0.0^{\circ}\right)$

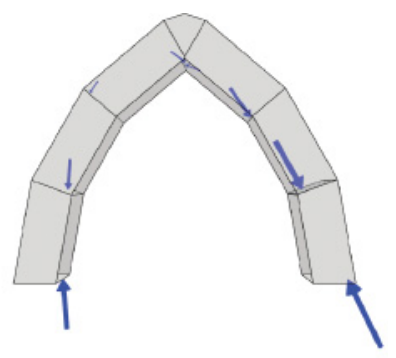

(b) Equilibrium $\left(17.4^{\circ}\right)$

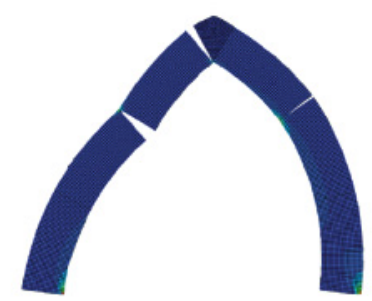

(d) FEM w Inequality $\left(16.8^{\circ}\right)$

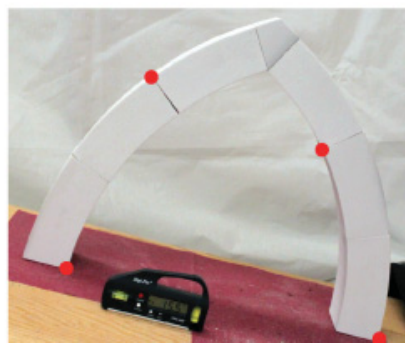

(f) Physical Model $\left(15.5^{\circ}\right)$
Fig. 8. The Gothic arch, at the critical tilt angle as predicted by (a) the inverse FEM and (b) the block equilibrium method, where the one possible set of contact forces is shown with arrows. (c) Continuum FEM predicts tension immediately after tilt. (e, f) The physical model fails at a critical angle slightly less than that predicted by (d) the inequality-constrained forward FEM, inverse FEM, and equilibrium method. Red dots indicate locations where hinges develop between blocks.

constraints. The FEM with inequality contact constraints performs better but fails to predict the stability of the minimum thickness stable arch, and for a thicker $\operatorname{arch}(t / R=0.150)$ predicts a critical angle over $10 \%$ smaller than the analytic solution (see Figure 7 ). Both the block equilibrium method and inverse FEM predict a critical angle equal to the analytic solution. The physical structure can fail the tilt test before the "theoretical critical angle" for several reasons, including slight variations in block size, imperfections in the constructed geometry due to fabrication error, wearing off of block corners from repeated experiments, or insufficient friction at the block interfaces. The printed arch failed at a surprisingly low angle of $4.7^{\circ}$, which corresponds to an effective thickness of $87 \%$ or $t / R=0.130$, most likely due to these factors.

Gothic Arches. Similar to the circular arch, the naive continuum FEM predicts failure as soon as the Gothic arch is tilted beyond the horizontal rest position. The FEM with inequality contact constraints predicts a higher critical angle, but slightly less than that predicted by the inverse FEM and the block equilibrium method. An analytical ground-truth value is not known, but we compare with results from the physical test. Similar to the circular arch example,

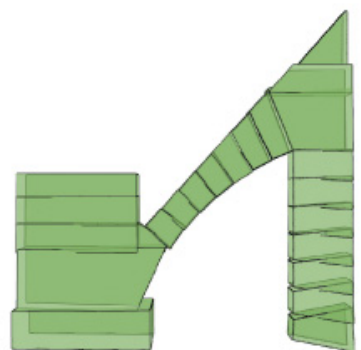

(a) Inverse FEM (5.7)

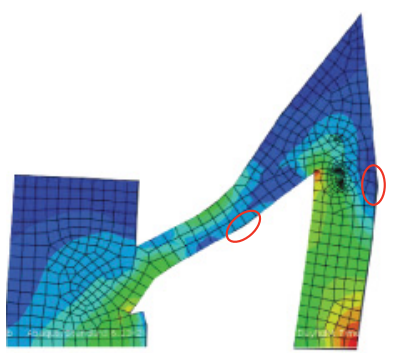

(c) Naïve FEM $\left(0.0^{\circ}\right)$

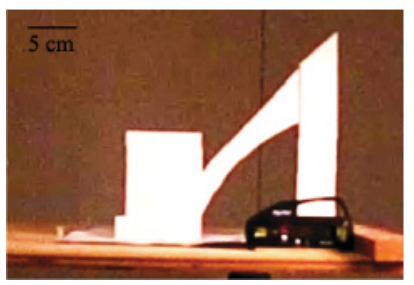

(e) Physical Model $\left(0.0^{\circ}\right)$

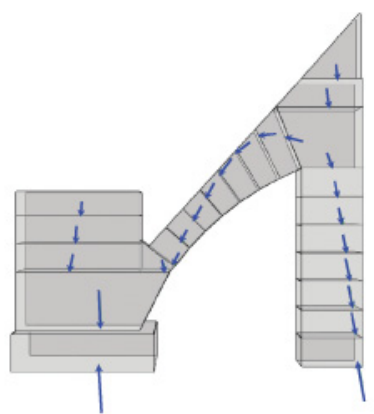

(b) Equilibrium $\left(5.7^{\circ}\right)$

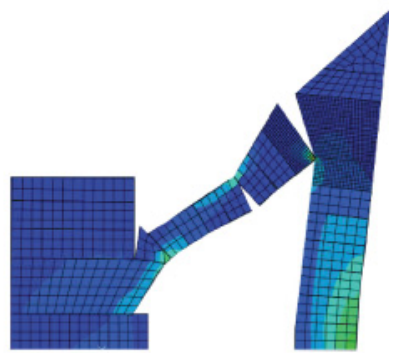

(d) FEM w Inequality $\left(0.0^{\circ}\right)$

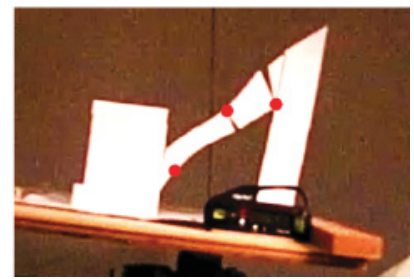

(f) Physical Model $\left(5.2^{\circ}\right)$
Fig. 9. The flying buttress, at the critical tilt angle as predicted by (a) the inverse FEM and (b) the block equilibrium method. (c) Continuum FEM and (d) inequality-constrained FEM. The latter two methods are overconservative, as validated by $(e, f)$ a physical tilt test.

the printed structure fails at a critical angle slightly below that predicted by the inverse FEM and equilibrium methods.

The Three-Legged $\pi$. As discussed in Section 5, the threelegged $\pi$ is a good didactic example, as it is a simple structure with static indeterminacy. The printed structure fails at a critical angle far above that predicted by the forward FEM methods, but slightly below that predicted by the inverse FEM and equilibrium methods (see Figure 6).

Flying Buttress. We compared critical angle predictions of the four numerical methods against a 3D-printed flying buttress model (see Figure 9). As with the $\pi$ model, the inverse FEM and block equilibrium predict the same critical angle, which was close to the experimental results. As expected, the naive FEM is overly conservative and predicts tension even at the rest position. More surprisingly, as in three-legged $\pi$, FEM with inequality contact constraints also fails significantly below the experimental or equilibrium/inverse FEM results. We observe that small deformations cause hinges to appear even at the rest orientation, and this geometric nonlinearity in the topology of the contacts likely leads to early failure. 


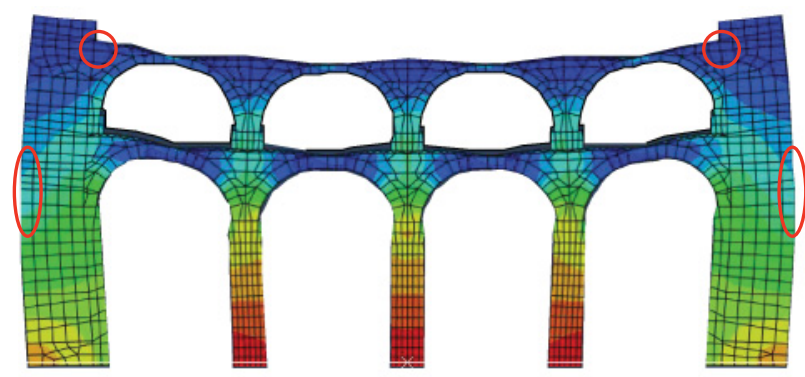

(a) Naïve FEM $\left(0.0^{\circ}\right)$

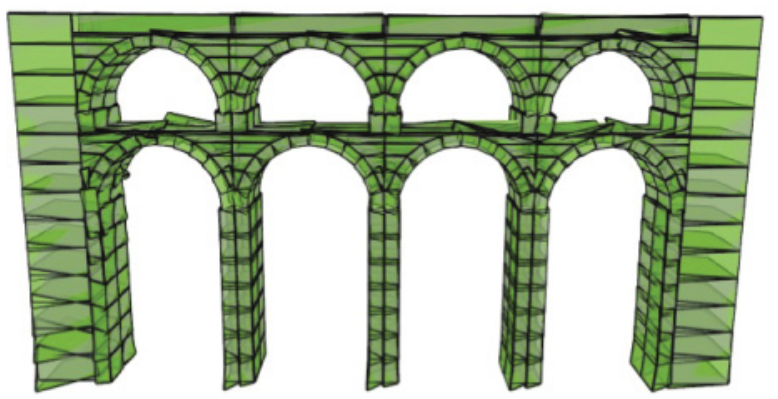

(c) Inverse FEM $\left(6.5^{\circ}\right)$

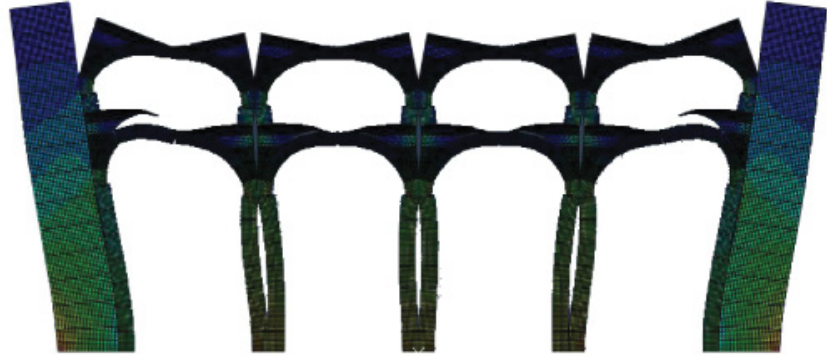

(b) FEM w Inequality $\left(0.0^{\circ}\right)^{*}$

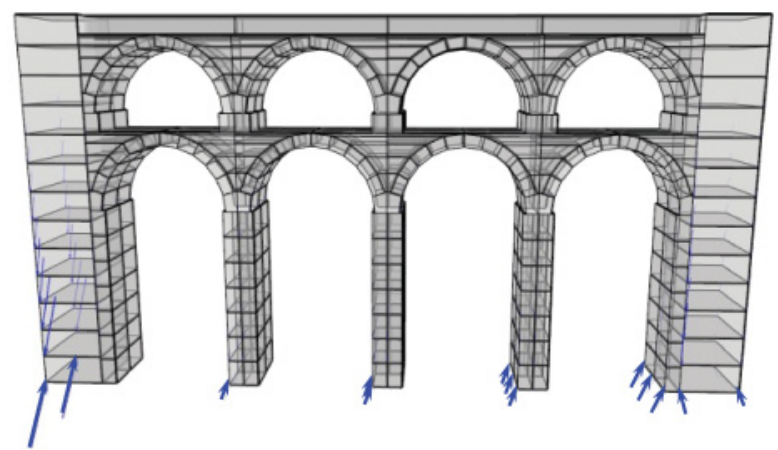

(d) Equilibrium $\left(6.5^{\circ}\right)$

Fig. 10. Tilt-test results for our aqueduct model, similar in spirit to the Roman Aqueduct of Segovia. (a) The stress distribution/displacement at the rest configuration predicted by naïve continuum FEM. Colors indicate magnitude of stress (red is higher, and blue is lower), and circled regions indicate areas of tension. (b) Inequality-constrained forward FEM fails to find an equilibrium solution even at the rest position. (c) The solved-for rest shape of the blocks predicted by the inverse FEM, and (d) one possible set of contact forces output by the block equilibrium method at the critical angle $\left(6.5^{\circ}\right)$.

* In (b), Abaqus fails to converge to a solution at $0.0^{\circ}$, but here we show the deformation at an intermediate increment stage.

Aqueduct. Scanned geometry for the Aqueduct of Segovia is unfortunately not available, so we analyzed the stability of a similar two-tier aqueduct we modeled ourselves (see Figure 10). Similar to the flying buttress, the naïve FEM outputs tension immediately, and the forward FEM with inequality contact constraints also fails to converge even at the rest position. The aqueduct is made of numerous blocks that constitute a complex topology of interfaces, exacerbating the problem of geometric nonlinearity. The inverse FEM and block equilibrium methods predict the same critical angle of $6.5^{\circ}$.

\section{DISCUSSION AND CONCLUSIONS}

This article examines two different traditions of methods used for static analysis of masonry: linear elastic analysis using FEM and equilibrium methods. The former is widely employed by practitioners across numerous fields of engineering, but when naïvely applied to masonry, it gives incorrect results. The latter approach is favored by many researchers as a simple but more reliable alternative for predicting whether or not a building stands. After examining these two classes of methods in detail, we draw several conclusions:

First, linear elastic finite element analysis with continuum equality constraints used to model interblock contacts (as is often done in practice) is wholly inadequate for predicting stability, as it is far too conservative - it cannot differentiate between safe and unsafe hinging modes, crucial to the analysis of all of the examples presented previously.

Replacing the contact constraints with one-sided constraints, to allow hinging, gives good results in some cases, particularly where the contact geometry of the blocks is simple (e.g., the arch and Gothic arch). However, in others, replacing the contact constraints alone still fails to give accurate results (the three-legged pi, flying buttress, and aqueduct). This is because interblock contact is geometrically nonlinear, even geometrically discontinuous: small deformations of the blocks can completely change the set of possible hinging modes. Elastic FEM resolves the problem of static indeterminacy by way of choosing one particular material model. Different ways of resolving the indeterminacy would lead to different deformations of the block, which leads to different admissible hinging modes.

Other culprits suggested in the literature for the error of forward FEM have included an insufficiently sophisticated material model, the extreme stiffness of stone, size of the elements, and so forth. It is true that an increasingly complex implementation of the FEM must eventually converge to a correct analysis of stability. However, the results are sensitive to the choice of parameters (which are not straightforward to tune even in commercial packages) and computationally expensive to obtain. On the other hand, inverse FEM and equilibrium methods are reliable even with a simple material model and elements as coarse as the structure's blocks. By fixing the deformed geometry, and consequently also the contact geometry, the inverse FEM and equilibrium methods both sidestep all of these difficulties, without incurring the complexity and computational cost of FEM with sophisticated contact and material models.

Indeed, we have shown that linear elastic analysis using finite elements, with a minor modification to solve the inverse rather than the forward problem, becomes a dual formulation of the block 
equilibrium method and gives the same, equally reliable results, reconciling the two traditions. The two classes of methods turn out to be two different ways of expressing an equivalent problem. That being said, the block equilibrium method is particularly elegant. Since it is formulated using force variables, it does not introduce counterintuitive (inverse) displacements, and it also includes the compression-only constraints directly (instead of formulating them indirectly in terms of displacements).

Limitations. We have made several assumptions about the behavior and modeling of masonry structures that could be relaxed to increase the sophistication of the stability analysis. Perhaps the biggest assumption we have made is that of infinite friction-a fully satisfactory theory combining equilibrium analysis with even a simple Coulomb friction model remains an elusive and extremely important goal in computational architecture. Infinitely thin hinges occurring at fixed locations are also an idealistic assumption violated in reality. For example, as we see in the arch and Gothic arch physical experiments, blocks' corners become rounded with wear, which affects the location of the hinges and the stability of the structures. We have also assumed that the blocks in the structure can support arbitrarily high internal tensile stress. This assumption is reasonable for small blocks in simple contact configurations, but not for large horizontal slabs like the one in our three-legged $\pi$ model. Finally, both the equilibrium and FEM results depend on the discretization of a structure into blocks, especially the configuration of interfaces. Finding the optimal level of discretization between the two extremes-modeling the structure as a monolith and modeling each block individually-is a difficult problem that must take into account practical cost and desired accuracy.

\section{APPENDIX}

\section{Relationship to Thrust Network Analysis (TNA)}

For a full explanation of Thrust Network Analysis, we refer to Block [2009]. Here, we focus on illustrating the relationship between TNA and the block equilibrium method and how FEM has been used within equilibrium analysis in the past.

TNA Versus Block Equilibrium Method. Every thrust network in equilibrium corresponds to a set of blocks in frictionless equilibrium. (The reverse is also true but only if the topology of the set of blocks is sufficiently simple, i.e., surface-like.) To see this, take the thrust network and create a set of blocks so that (1) every vertex of the thrust network is the center of mass of one block, (2) the mass of each block is equal to the mass of the vertex, (3) two blocks meet at an interface if and only if the corresponding vertices share an edge, and (4) the interface between blocks is perpendicular to the thrust network edge.

One construction is to first compute the intrinsic dual of the thrust network mesh. The dual cells will satisfy properties (3) and (4) automatically, and can be "fattened" in the normal direction of the faces to create blocks. Fattening arbitrarily will make the blocks have the wrong total mass and center of mass. To fix this, add or remove material from each block (while keeping it in touch with its neighbors) until (1) and (2) are satisfied; we can always do this by making some parts of the block thinner and other parts thicker. Now for each edge $e_{\mathrm{ij}}$ of the thrust network with weight $w_{\mathrm{ij}}$, add a contact force $w_{\mathrm{ij}} e_{\mathrm{ij}}$ at the point where $e_{\mathrm{ij}}$ intersects the interface between blocks $i$ and $j$. (We can also distribute this force to the interface corners.) Since this force is parallel to the torque arm, it exerts no torque on block $i$ or $j$. Moreover, the total force on the center of mass of block $i$ is $F_{g}+\Sigma_{j \sim i} w_{\mathrm{ij}} e_{\mathrm{ij}}$, where $F_{g}$ is the gravitational force, and the sum is over neighboring blocks of $i$. This is equal to zero since this is also the equation of thrust network equilibrium.

So, our proof in Section 6 also holds for thrust networks: every thrust network in equilibrium corresponds to some set of blocks (constructed as described earlier) that are stable under inverse FEM.

TNA Versus FEM. Fraternali [2010] has shown that the edge weights in a thrust network can be interpreted as gradient jumps in a finite element discretization of the polyhedral Airy stress function, discretized using piecewise linear elements. However, there are two key differences between this approach and the FEM (direct stiffness method) we consider in our work: (1) Analyzing stability via the Airy stress function still falls into the equilibrium school of analysis since it treats the geometry of the structure as static and then computes whether a stress function exists that satisfies the equilibrium. There is no deformation involved. While the unknown Airy stress function is found using FEM in the broader sense of the term (as a numerical method to solve arbitrary PDEs), this is different from the direct stiffness method that we consider in our work. (2) The work based on discretizing Airy stress analyzes only infinitesimally thin shells and doesn't apply to volumetric blocks.

\section{Linearization of Inverse FEM}

In the linear formulations of elasticity used earlier for both forward and inverse FEM, we assume that the block deformation $\mathbf{u}=\mathbf{x}_{\mathrm{aft}}-$ $\mathbf{x}_{\mathrm{bfr}}$ is small (but not negligible), but that $\|\mathbf{u}\|^{2}$ is even smaller and can be neglected [Mase et al. 2009]. Here we show that $\tilde{\mathbf{K}}$ is the correct linearization of the forces arising in the inverse FEM formulation described in Equation (17). We first review how the forces are linearized in forward FEM, and then extend the analysis to inverse FEM.

Consider the full nonlinear elastic energy $U\left(\mathbf{x}_{\mathrm{aft}}, \mathbf{x}_{\mathrm{bfr}}\right)$, and Taylor expand it about $\mathbf{x}_{\mathrm{aft}}=\mathbf{x}_{\mathrm{bfr}}$ :

$$
\begin{aligned}
U\left(\mathbf{x}_{\mathrm{bfr}}+\mathbf{u}, \mathbf{x}_{\mathrm{bfr}}\right)= & U\left(\mathbf{x}_{\mathrm{bfr}}, \mathbf{x}_{\mathrm{bfr}}\right)+\frac{\partial U}{\partial \mathbf{x}_{\mathrm{aft}}}\left(\mathbf{x}_{\mathrm{bfr}}, \mathbf{x}_{\mathrm{bfr}}\right) \mathbf{u} \\
& +\frac{1}{2} \mathbf{u}^{\top} \frac{\partial^{2} U}{\partial \mathbf{x}_{\mathrm{aft}}^{2}}\left(\mathbf{x}_{\mathrm{bfr}}, \mathbf{x}_{\mathrm{bfr}}\right) \mathbf{u}+o\left(\|\mathbf{u}\|^{3}\right) .
\end{aligned}
$$

The first two terms vanish since $U$ is minimized when $\mathbf{x}_{\mathrm{aft}}=\mathbf{x}_{\mathrm{bfr}}$. The force expressed in displacement degrees of freedom is then:

$$
\frac{d U\left(\mathbf{x}_{\mathrm{bfr}}+\mathbf{u}, \mathbf{x}_{\mathrm{bfr}}\right)}{d \mathbf{u}}=\frac{\partial^{2} U}{\partial \mathbf{x}_{\mathrm{aft}}^{2}}\left(\mathbf{x}_{\mathrm{bfr}}, \mathbf{x}_{\mathrm{bfr}}\right) \mathbf{u}+o\left(\|\mathbf{u}\|^{2}\right),
$$

and $\mathbf{K}=\partial^{2} U / \partial \mathbf{x}_{\mathrm{aft}}^{2}\left(\mathbf{x}_{\mathrm{bfr}}, \mathbf{x}_{\mathrm{bfr}}\right)$ is constant with respect to $\mathbf{x}_{\mathrm{aft}}$. This linearization is used in the elastic energy term in Equation (8).

In the case of inverse FEM, $\mathbf{x}_{\mathrm{bfr}}$ is the unknown variable. We thus take the Taylor expansion about $\mathbf{x}_{\mathrm{bfr}}=\mathbf{x}_{\mathrm{aft}}$ :

$$
\begin{aligned}
U\left(\mathbf{x}_{\mathrm{aft}}, \mathbf{x}_{\mathrm{aft}}-\mathbf{u}\right)= & U\left(\mathbf{x}_{\mathrm{aft}}, \mathbf{x}_{\mathrm{aft}}\right)-\frac{\partial U}{\partial \mathbf{x}_{\mathrm{bfr}}}\left(\mathbf{x}_{\mathrm{aft}}, \mathbf{x}_{\mathrm{aft}}\right) \mathbf{u} \\
& +\frac{1}{2} \mathbf{u}^{\top} \frac{\partial^{2} U}{\partial \mathbf{x}_{\mathrm{bfr}}^{2}}\left(\mathbf{x}_{\mathrm{aft}}, \mathbf{x}_{\mathrm{aft}}\right) \mathbf{u}+o\left(\|\mathbf{u}\|^{3}\right) .
\end{aligned}
$$

Once again, the first two terms are zero since $U$ has a local minimum at $\mathbf{x}_{\mathrm{bfr}}=\mathbf{x}_{\mathrm{aft}}$. The force with respect to displacement is

$$
\frac{d U\left(\mathbf{x}_{\mathrm{aft}}, \mathbf{x}_{\mathrm{aft}}-\mathbf{u}\right)}{d \mathbf{u}}=\frac{\partial^{2} U}{\partial \mathbf{x}_{\mathrm{bfr}}^{2}}\left(\mathbf{x}_{\mathrm{aft}}, \mathbf{x}_{\mathrm{aft}}\right) \mathbf{u}+o\left(\|\mathbf{u}\|^{2}\right) .
$$

Finally, we show that $\frac{\partial^{2} U}{\partial \mathbf{x}_{\mathrm{bfr}}^{2}}\left(\mathbf{x}_{\mathrm{aft}}, \mathbf{x}_{\mathrm{aft}}\right)=\frac{\partial^{2} U}{\partial \mathbf{x}_{\mathrm{aft}}^{2}}\left(\mathbf{x}_{\mathrm{aft}}, \mathbf{x}_{\mathrm{aft}}\right)=\tilde{\mathbf{K}}$, the stiffness matrix in Equation (17). To see this, notice that $\frac{\partial U}{\partial \mathbf{x}_{\text {aft }}}(\mathbf{x}, \mathbf{x})$ is 
zero (thus constant) for any $\mathbf{x}$, since $U$ has a minimum at $\mathbf{x}_{\mathrm{bfr}}=\mathbf{x}_{\mathrm{aft}}$. Therefore,

$$
0=\frac{d}{d \mathbf{x}} \frac{\partial U}{\partial \mathbf{x}_{\mathrm{aft}}}(\mathbf{x}, \mathbf{x})=\frac{\partial^{2} U}{\partial \mathbf{x}_{\mathrm{aft}}^{2}}(\mathbf{x}, \mathbf{x})+\frac{\partial^{2} U}{\partial \mathbf{x}_{\mathrm{bfr}} \partial \mathbf{x}_{\mathrm{aft}}}(\mathbf{x}, \mathbf{x}),
$$

and by an identical argument,

$$
0=\frac{d}{d \mathbf{x}} \frac{\partial U}{\partial \mathbf{x}_{\mathrm{bfr}}}(\mathbf{x}, \mathbf{x})=\frac{\partial^{2} U}{\partial \mathbf{x}_{\mathrm{bfr}}^{2}}(\mathbf{x}, \mathbf{x})+\frac{\partial^{2} U}{\partial \mathbf{x}_{\mathrm{aft}} \partial \mathbf{x}_{\mathrm{bfr}}}(\mathbf{x}, \mathbf{x}) .
$$

Combining the previous two equations and using equality of mixed partials yields

$$
\frac{\partial^{2} U}{\partial \mathbf{x}_{\mathrm{bfr}}^{2}}(\mathbf{x}, \mathbf{x})=\frac{\partial^{2} U}{\partial \mathbf{x}_{\mathrm{aft}}^{2}}(\mathbf{x}, \mathbf{x})
$$

for any $\mathbf{x}$ (and in particular, $\mathbf{x}=\mathbf{x}_{\mathrm{aft}}$ ).

\section{Equivalence of the Inverse FEM and Equilibrium Methods (Proof Continued from Section 6)}

Torques induced by the displacements solved for using the inverse FEM also satisfy the constraints of the block equilibrium method. Consider a single block and its full nonlinear elastic energy, $U\left(\mathbf{x}_{\mathrm{aft}}, \mathbf{x}_{\mathrm{bfr}}\right)$, where $\mathbf{x}_{\mathrm{aft}}$ and $\mathbf{x}_{\mathrm{bfr}}$ are expressed in coordinates with the origin at the block's center of mass. Let $\mathbf{w}$ be a unit vector and $[\mathbf{w}]$ be the cross-product matrix of $\mathbf{w}$; then, the matrix $\mathbf{R}_{\mathbf{w}}(t)=e^{[\mathbf{w}] t}$ represents rotation about the center of mass by $t$ radians counterclockwise around the $\mathbf{w}$ axis. Since elastic energy is invariant under transformation of $\mathbf{x}_{\mathrm{aft}}$ by rigid motions,

$$
U\left(\mathbf{R}_{\mathbf{w}}(t) \mathbf{x}_{\mathrm{aft}}, \mathbf{x}_{\mathrm{bfr}}\right)=U\left(\mathbf{x}_{\mathrm{aft}}, \mathbf{x}_{\mathrm{bfr}}\right)
$$

for any $t$. Differentiating with respect to $t$ gives

$$
0=\frac{d \mathbf{R}_{\mathbf{w}}}{d t}(t) \sum_{i} \frac{\partial U}{\partial \mathbf{x}_{\mathrm{aft}}^{i}}\left(\mathbf{R}_{\mathrm{w}}(t) \mathbf{x}_{\mathrm{aft}}, \mathbf{x}_{\mathrm{bfr}}\right) .
$$

Since $\frac{d \mathbf{R}_{\mathbf{w}}}{d t}(0)=[\mathbf{w}]$, evaluating the previous at $t=0$ gives

$$
0=[\mathbf{w}] \sum_{i} \frac{\partial U}{\partial \mathbf{x}_{\mathrm{aft}}^{i}}\left(\mathbf{x}_{\mathrm{aft}}, \mathbf{x}_{\mathrm{bfr}}\right)
$$

or, since $\mathbf{f}_{\mathrm{in}}^{i}=-\frac{\partial U}{\partial \mathbf{x}_{\mathrm{aft}}^{i}}$,

$$
0=[\mathbf{w}] \sum_{i} \mathbf{f}_{\mathrm{in}}^{i}\left(\mathbf{x}_{\mathrm{aft}}, \mathbf{x}_{\mathrm{bfr}}\right) .
$$

Since $\mathbf{w}$ is arbitrary, the full net nonlinear internal force exerts no torque on the block. We can see that the same must hold for the linearized forces. Taylor expand $U$ about $\mathbf{x}_{\mathrm{bfr}}$ to get

$$
U\left(\mathbf{x}_{\mathrm{bfr}}+\mathbf{u}, \mathbf{x}_{\mathrm{bfr}}\right)=\sum_{i} \frac{\partial U}{\partial \mathbf{x}_{\mathrm{aft}}^{i}}\left(\mathbf{x}_{\mathrm{bfr}}, \mathbf{x}_{\mathrm{bfr}}\right) \mathbf{u}^{i}+o\left(\|\mathbf{u}\|^{2}\right)
$$

and

$$
0=-[\mathbf{W}] \mathbf{K}\left(\mathbf{x}_{\mathrm{bfr}}\right) \mathbf{u}+o\left(\|\mathbf{u}\|^{2}\right),
$$

where $\mathbf{u}=\mathbf{x}_{\mathrm{aft}}-\mathbf{x}_{\mathrm{bfr}}$ and [W] is a block-diagonal matrix with blocks $[\mathbf{w}]$. The equation holds for all $\mathbf{u}$, so differentiating and setting $\mathbf{u}=0$ yields $0=[\mathbf{W}] \mathbf{K}$, and so

$$
0=[\mathbf{W}] \mathbf{K u}=\sum_{i}[\mathbf{w}] \mathbf{f}_{\mathrm{in}}^{i}
$$

for any $\mathbf{u}$, where $\mathbf{f}_{\text {in }}^{i}$ are now linearized forces. It follows from Equation (19) that $0=[\mathbf{w}] \sum_{i}\left(\mathbf{f}_{\mathrm{w}}^{i}+\mathbf{f}_{\mathrm{ct}}^{i}\right)$, which is the torque balance of the block.

\section{Experiment Parameters and Data}

Table V describes the parameter setting for Abaqus simulations, and Table VI shows the complete results from the physical tilt test.

Table V. Abaqus/Standard Settings

\begin{tabular}{lc}
\hline Young's modulus & $1.0 \times 10^{6}$ \\
Poisson's ratio & 0.2 \\
Density & 10.0 \\
Mesh/element type & CPS4R \\
Contact relationship & Hard \\
Friction penalty* & coeff $=100$ \\
\hline *We experimented with larger values of coeff as well as the \\
"Rough" contact formulation. We also experimented with. \\
different increment sizes and toggling NLGEOM option. We \\
report the least conservative result.
\end{tabular}

Table VI. Physical Tilt-Test Results

\begin{tabular}{c|rrrc}
\hline Model & \multicolumn{1}{|c}{1} & \multicolumn{1}{c}{2} & \multicolumn{1}{c}{3} & Average \\
\hline Cube & 41.3 & 40.8 & 40.9 & 41.0 \\
Arch, thick & 4.8 & 4.8 & 4.5 & 4.7 \\
Gothic arch & 15.8 & 15.7 & 15.5 & 15.7 \\
3-legged $\pi$ & 14.3 & 14.5 & 14.2 & 14.3 \\
Flying buttress & 5.2 & 4.3 & 3.8 & 4.4 \\
\hline
\end{tabular}

In the naive FEM, we model a structure as a single continuous part. In contrast, in the FEM with inequality contact constraints, each block composing a structure is modeled as a separate part, and the interaction between the blocks is defined through tangential and normal contact properties: a high coefficient of friction to prevent sliding and a hard contact to disallow interpenetration but allow separation.

\section{Scaling Factors for Figures}

Table VII lists the scaling factors used in the figures to visualize displacements.

Table VII. Displacements Scale Factor To aid visualization, the displacements in the figures of this article have been exaggerated.

\begin{tabular}{rc}
\hline Figure & Factor \\
\hline $6(\mathrm{c})$ & $6.4 \times 10^{2}$ \\
$7(\mathrm{e})$ & $7.5 \times 10^{2}$ \\
$7(\mathrm{f})$ & $2.1 \times 10$ \\
$8(\mathrm{c})$ & $1.0 \times 10^{2}$ \\
$8(\mathrm{~d})$ & $5.0 \times 10^{1}$ \\
$9(\mathrm{c})$ & $5.1 \times 10^{2}$ \\
$9(\mathrm{~d})$ & $2.1 \times 10^{2}$ \\
$10(\mathrm{a})$ & $8.0 \times 10^{2}$ \\
$10(\mathrm{~b})$ & $2.0 \times 10^{5}$ \\
\hline
\end{tabular}

\section{ACKNOWLEDGMENTS}

We would like to thank William Plunkett for his assistance with the physical experiments. We are grateful to Sylvain Paris, David Levin, Shinjiro Sueda, Desai Chen, and Danny Kaufman for their insightful feedback and discussions. We also thank Zhandos Orazalin for his help with Abaqus software.

\section{REFERENCES}

Autodesk. 2014. Robot Structural Analysis Professional. (November 2014). http://www.autodesk.com/products/robot-structural-analysis/overview. 
David Baraff. 1993. Non-penetrating rigid body simulation. In Eurographics 93 State of the Art Report, J. Fagerberg, D. C. Mowery, and R. R. Nelson (Eds.). Eurographics Association.

Klaus-Jürgen Bathe. 2006. Finite Element Procedures. Klaus-Jurgen Bathe. Philippe Block, Thierry Ciblac, and John Ochsendorf. 2006. Real-time limit analysis of vaulted masonry buildings. Computers \& Structures 84, 29 (2006), 1841-1852.

Philippe Block and John Ochsendorf. 2007. Thrust network analysis: A new methodology for three-dimensional equilibrium. Journal of the International Association for Shell and Spatial Structures 48, 3 (2007), 167-173.

Philippe Philippe Camille Vincent Block. 2009. Thrust Network Analysis: Exploring Three-Dimensional Equilibrium. Ph.D. Dissertation. Massachusetts Institute of Technology.

Sandro Brasile, Raffaele Casciaro, and Giovanni Formica. 2007. Multilevel approach for brick masonry walls-Part I: A numerical strategy for the nonlinear analysis. Computer Methods in Applied Mechanics and Engineering 196, 49 (2007), 4934-4951.

Jaime Cervera Bravo, Jose Ignacio Hernando Garcia, and Juan Francisco De La Torre Calvo. 1994. Acueducto de Segovia Comportamiento Mecanico. Technical Report. Universidad Politecnica de Madrid.

Philip Brune and Renato Perucchio. 2012. Roman concrete vaulting in the great hall of Trajans markets: Structural evaluation. Journal of Architectural Engineering 18, 4 (2012), 332-340.

Xiang Chen, Changxi Zheng, Weiwei Xu, and Kun Zhou. 2014. An asymptotic numerical method for inverse elastic shape design. ACM Transactions on Graphics (In Proceedings of SIGGRAPH 2014) 33, 4 (Aug. 2014).

Computers and Structures, Inc. 2014. SAP2000. (June 2014). http://www. csiamerica.com/products/sap2000.

Stelian Coros, Sebastian Martin, Bernhard Thomaszewski, Christian Schumacher, Robert Sumner, and Markus Gross. 2012. Deformable objects alive! ACM Transactions on Graphics 31, 4, Article 69 (July 2012), 9 pages. DOI : http://dx.doi.org/10.1145/2185520.2185565

P. A. Cundall. 1971. A computer model for simulating progressive, largescale movements in blocky rock systems. In Proceedings of the Symposium of the International Society of Rock Mechanics. Vol. 2. Nancy.

Dassault Système. 2014. Abaqus Unified FEA. (June 2014). http://www.3ds. com/products-services/simulia/portfolio/abaqus/overview/.

Fernando de Goes, Pierre Alliez, Houman Owhadi, Mathieu Desbrun, and others. 2013. On the equilibrium of simplicial masonry structures. ACM Transactions on Graphics 32, 4 (2013), 93:1-93:10.

Matthew J. DeJong. 2009. Seismic Assessment Strategies for Masonry Structures. Ph.D. Dissertation. Massachusetts Institute of Technology, Department of Architecture.

Mario Deuss, Daniele Panozzo, Emily Whiting, Yang Liu, Philippe Block, Olga Sorkine-Hornung, and Mark Pauly. 2014. Assembling selfsupporting structures. ACM Transactions on Graphics (SIGGRAPH ASIA) (2014).

John Dolbow and T. Belytschko. 1999. A finite element method for crack growth without remeshing. International Journal of Numerical Methods in Engineering 46, 1 (1999), 131-150.

Kenny Erleben. 2007. Velocity-based shock propagation for multibody dynamics animation. ACM Transactions on Graphics (TOG) 26, 2 (2007), 12.

Fernando Fraternali. 2010. A thrust network approach to the equilibrium problem of unreinforced masonry vaults via polyhedral stress functions. Mechanics Research Communications 37, 2 (2010), 198-204.

Eran Guendelman, Robert Bridson, and Ronald Fedkiw. 2003. Nonconvex rigid bodies with stacking. In ACM Transactions on Graphics (TOG), Vol. 22. ACM, 871-878.
John C. Hart, Brent Baker, and Jeyprakash Michaelraj. 2003. Structural simulation of tree growth and response. The Visual Computer 19, 2-3 (2003), 151-163.

Jacques Heyman. 1966. The stone skeleton. International Journal of Solids and Structures 2 (1966), 249-279.

Jacques Heyman. 1995. The Stone Skeleton: Structural Engineering of Masonry Architecture. Cambridge University Press.

Jacques Heyman. 1996. Elements of the Theory of Structures. Cambridge University Press.

Shu-Wei Hsu and John Keyser. 2012. Automated constraint placement to maintain pile shape. ACM Transactions on Graphics (TOG) 31, 6 (2012), 150

Santiago Huerta. 2001. Mechanics of masonry vaults: The equilibrium approach. Universidade do Minho (2001).

Santiago Huerta. 2008. The analysis of masonry architecture: A historical approach: To the memory of professor Henry J. Cowan. Architectural Science Review 51, 4 (2008), 297-328.

Danny M. Kaufman, Shinjiro Sueda, Doug L. James, and Dinesh K. Pai. 2008. Staggered projections for frictional contact in multibody systems. In ACM Transactions on Graphics (TOG), Vol. 27. ACM, 164.

Yang Liu, Hao Pan, John Snyder, Wenping Wang, and Baining Guo. 2013. Computing self-supporting surfaces by regular triangulation. ACM Transactions on Graphics 32, 4 (July 2013), 92.

R. K. Livesley. 1978. Limit analysis of structures formed from rigid blocks. International Journal of Numerical Methods in Engineering 12 (1978), 1853-1871.

Paulo B. Lourenço, Jan G. Rots, and Johan Blaauwendraad. 1998. Continuum model for masonry: Parameter estimation and validation. Journal of Structural Engineering 124, 6 (1998), 642-652.

Robert Mark. 1982. Experiments in Gothic Structure. Vol. 11. MIT Press, Cambridge, MA.

Sebastian Martin, Peter Kaufmann, Mario Botsch, Eitan Grinspun, and Markus Gross. 2010. Unified simulation of elastic rods, shells, and solids. ACM Transactions on Graphics 29, 4 (2010), 39:1-39:10.

G. Thomas Mase, Ronald E. Smelser, and George E. Mase. 2009. Continuum Mechanics for Engineers. CRC Press.

Matthias Müller, Jos Stam, Doug James, and Nils Thürey. 2008. Real time physics: Class notes. In ACM SIGGRAPH 2008 classes. ACM, 88.

Rahul Narain, Abhinav Golas, and Ming C. Lin. 2010. Free-flowing granular materials with two-way solid coupling. In ACM Transactions on Graphics (TOG), Vol. 29. ACM, 173.

John Ochsendorf. 2002. Collapse of Masonry Structures. Ph.D. Dissertation. University of Cambridge.

Paul Painlevé. 1895. Sur les lois du frottement de glissement. Comptes Rendus Academie des Sciences Paris 121, 112-115.

Daniele Panozzo, Philippe Block, and Olga Sorkine-Hornung. 2013. Designing unreinforced masonry models. ACM Transactions on Graphics (In Proceedings of ACM SIGGRAPH) 32, 4 (2013), 91:1-91:12.

Romain Prévost, Emily Whiting, Sylvain Lefebvre, and Olga SorkineHornung. 2013. Make it stand: Balancing shapes for 3D fabrication. ACM Transactions on Graphics (In Proceedings of ACM SIGGRAPH) 32, 4 (2013), 81:1-81:10.

Pere Roca, Miguel Cervera, Giuseppe Gariup, and others. 2010. Structural analysis of masonry historical constructions. Classical and advanced approaches. Archives of Computational Methods in Engineering 17, 3 (2010), 299-325.

Xiaohan Shi, Kun Zhou, Yiying Tong, Mathieu Desbrun, Hujun Bao, and Baining Guo. 2007. Mesh puppetry: Cascading optimization of mesh deformation with inverse kinematics. ACM Transactions on Graphics 26, 3 (2007), 81. http://doi.acm.org/10.1145/1276377.1276479. 
Eftychios Sifakis and Jernej Barbic. 2012. FEM simulation of 3D deformable solids: A practitioner's guide to theory, discretization and model reduction. In ACM SIGGRAPH 2012 Courses. ACM, 20.

Jeffrey Smith, Jessica K. Hodgins, Irving Oppenheim, and Andrew Witkin. 2002. Creating models of truss structures with optimization. In Proceedings of SIGGRAPH 2002 (Computer Graphics Proceedings, Annual Conference Series), John Hughes (Ed.). ACM, ACM Press / ACM SIGGRAPH, 295-301.

Ondrej Stava, Juraj Vanek, Bedrich Benes, Nathan Carr, and Radomír Měch. 2012. Stress relief: Improving structural strength of 3D printable objects. ACM Transactions on Graphics 31, 4, Article 48 (July 2012), 11 pages. DOI : http://dx.doi.org/10.1145/2185520.2185544

Demetri Terzopoulos and Kurt Fleischer. 1988. Modeling inelastic deformation: Viscolelasticity, plasticity, fracture. SIGGRAPH Computer Graphics 22, 4 (June 1988), 269-278. DOI: http://dx.doi.org/10.1145/ 378456.378522

Demetri Terzopoulos, John Platt, Alan Barr, and Kurt Fleischer. 1987. Elastically deformable models. SIGGRAPH Computer Graphics 21, 4 (Aug. 1987), 205-214. DOI : http://dx.doi.org/10.1145/37402.37427

Nobuyuki Umetani, Takeo Igarashi, and Niloy J. Mitra. 2012. Guided exploration of physically valid shapes for furniture design. ACM Transactions on Graphics 31, 4 (2012), 86.

Nobuyuki Umetani, Kenshi Takayama, Jun Mitani, and Takeo Igarashi. 2010. Responsive FEM for aiding interactive geometric modeling. IEEE Computer Graphics and Applications 99 (2010), 43-53.
E. Vouga, Mathias Höbinger, Johannes Wallner, and Helmut Pottmann. 2012. Design of self-supporting surfaces. ACM Transactions on Graphics 31, 3 (2012), 87:1-87:11.

Emily Whiting, John Ochsendorf, and Frédo Durand. 2009. Procedural modeling of structurally-sound masonry buildings. ACM Transactions on Graphics 28, 5 (2009), 112.

Emily Whiting, Hijung Shin, Robert Wang, John Ochsendorf, and Frédo Durand. 2012. Structural optimization of 3D masonry buildings. ACM Transactions on Graphics 31, 6 (2012), 159:1-159:11.

Emily Jing Wei Whiting. 2012. Design of Structurally-Sound Masonry Buildings Using 3D Static Analysis. Ph.D. Dissertation. Massachusetts Institute of Technology.

Weiwei Xu, Jun Wang, KangKang Yin, Kun Zhou, Michiel van de Panne, Falai Chen, and Baining Guo. 2009. Joint-aware manipulation of deformable models. ACM Transactions on Graphics 28, 3, Article 35 (July 2009), 9 pages. DOI : http://dx.doi.org/10.1145/1531326.1531341

Jennifer Furstenau Zessin. 2012. Collapse Analysis of Unreinforced Masonry Domes and Curving Walls. Ph.D. Dissertation. Massachusetts Institute of Technology.

Qingnan Zhou, Julian Panetta, and Denis Zorin. 2013. Worst-case structural analysis. ACM Transactions on Graphics 32, 4, Article 137 (July 2013), 12 pages. DOI : http://dx.doi.org/10.1145/2461912.2461967

Received November 2014; revised August 2015; accepted October 2015 